Discussion Paper No. 08-103

\title{
Beschäftigungsstabilität im
} Konjunkturzyklus -

Eine empirische Analyse mit Individualdaten für Westdeutschland 1984-2005

Olaf Hübler und Thomas Walter

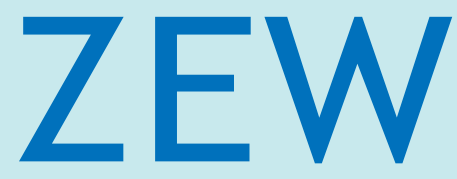

Zentrum für Europäische Wirtschaftsforschung $\mathrm{GmbH}$ Centre for European Economic Research 
Discussion Paper No. 08-103

\section{Beschäftigungsstabilität im Konjunkturzyklus - Eine empirische Analyse mit Individual- daten für Westdeutschland 1984-2005}

Olaf Hübler und Thomas Walter

Download this ZEW Discussion Paper from our ftp server:

ftp://ftp.zew.de/pub/zew-docs/dp/dp08103.pdf

Die Discussion Papers dienen einer möglichst schnellen Verbreitung von neueren Forschungsarbeiten des ZEW. Die Beiträge liegen in alleiniger Verantwortung der Autoren und stellen nicht notwendigerweise die Meinung des ZEW dar.

Discussion Papers are intended to make results of ZEW research promptly available to other economists in order to encourage discussion and suggestions for revisions. The authors are solely responsible for the contents which do not necessarily represent the opinion of the ZEW. 


\section{Das Wichtigste in Kürze}

Die Entwicklung der Beschäftigungsstabilität ist seit einiger Zeit vermehrt Gegenstand arbeitsökonomischer Analysen. Empirische Studien, die eine Veränderung der Beschäftigungsstabilität im Zeitverlauf untersuchen, liefern allerdings keine eindeutigen Ergebnisse. Einige Untersuchungen berichten von einer mit der Zeit abnehmenden Stabilität, andere erkennen nahezu keine Veränderungen. Wiederum andere Untersuchungen ermitteln eine zunehmende Beschäftigungsstabilität. Veränderungen der Beschäftigungsstabilität müssen jedoch keinem langfristigen Trend folgen, sondern können auch konjunkturbedingten Schwankungen unterliegen. Dies ist der Fall, wenn sich Mobilitätsprozesse auf dem Arbeitsmarkt während wirtschaftlicher Auf- und Abschwünge unterscheiden. Hierzu existieren zwar einige wenige empirische Untersuchungen, allerdings beruhen diese überwiegend auf aggregierten Daten. Mit solchen Daten lassen sich häufig lediglich Unterschiede in der Beschäftigungsstabilität während verschiedener konjunktureller Phasen erkennen, aber es lässt sich nicht ermitteln, ob die Unterschiede tatsächlich auf den Konjunkturverlauf oder auf andere Einflussgrößen zurückzuführen sind, die ebenfalls zyklischen Schwankungen unterliegen. In dieser Studie wird daher mit dem Sozio-ökonomischen Panel ein mikroökonomischer Datensatz eingesetzt, um so eine differenziertere Analyse des Einflusses konjunktureller Schwankungen auf die Stabilität von Beschäftigungsverhältnissen zu ermöglichen. Die individuelle Betriebszugehörigkeitsdauer wird dabei als zentrales Maß für die Stabilität eines Beschäftigungsverhältnisses herangezogen. Untersucht werden Beschäftigungsverhältnisse in Westdeutschland für den Zeitraum von 1984 bis 2005.

Die Ergebnisse zeigen, dass sich sowohl für Frauen als auch für Männer ein Einfluss der konjunkturellen Entwicklung auf die Beschäftigungsstabilität erkennen lässt. Die Beschäftigungsstabilität nimmt für beide Geschlechter zu Beginn eines wirtschaftlichen Aufschwungs zu. Mit zunehmender Dauer des Aufschwungs setzt allerdings für Männer ein entgegengesetzter Einfluss ein und das Risiko einer Beschäftigungsbeendigung steigt aufgrund der hohen Wachstumsraten der Vorperioden. Während eines Abschwunges nimmt die Beschäftigungsstabilität für beide Geschlechter ab. Aber auch hier zeigt sich nach einiger Zeit eine Abmilderung des Effektes für Männer. 


\section{Non-technical summary}

In recent years, the long-run development of job stability has been of considerable interest in empirical economic research resulting in a large number of published studies. However, these studies do not deliver a clear result. Some studies report on a decline of job stability, whereas other studies do not find any change or describe an increase in job security. These ambiguous findings suggest that job stability does not necessarily follow a long-run trend, but could also be subject to cyclical effects if job mobility is different in economic up- and downturns. Studies that try to investigate the impact of the business cycle on job stability are rare and mostly rely on aggregate data. Yet, with this type of data only differences in job stability during booms and recessions can be described, but it is not possible to establish a causal relationship. Therefore, we use micro-level data of the GSOEP to estimate the effect of the economic cycle on job stability in West Germany over the period from 1984 to 2005. We use individual tenure to measure job stability.

Our results show that the business cycle has a significant impact on job durations. In economic upturns job stability of men and women rises. However, with increasing duration of the upturn men experience a reverse effect and the risk of job termination starts to rise due to the high growth rates of the early upturn periods. During a recession job stability declines for both men and women. Similarly to the upturn, the effect for men is attenuated after some duration of the downturn. 


\title{
Beschäftigungsstabilität im Konjunkturzyklus - Eine empirische Analyse mit Individualdaten für Westdeutschland $1984-2005$
}

\author{
Olaf Hübler und Thomas Walter
}

\begin{abstract}
:
Der Beitrag untersucht unter Verwendung der Daten des Sozio-ökonomischen Panels für Westdeutschland über den Zeitraum 1984-2005, ob und in welcher Form die individuelle Betriebszugehörigkeitsdauer von konjunkturellen Schwankungen beeinflusst wird. Als Analyseinstrument dient die parametrische Zeitdaueranalyse, wobei Weibull- und loglogistische Accelerated Failure Time-Modelle herangezogen werden. Festzuhalten ist, dass sich sowohl für Frauen als auch für Männer ein Einfluss der konjunkturellen Entwicklung auf die Beschäftigungsstabilität erkennen lässt. Die Beschäftigungsstabilität nimmt für beide Geschlechter zu Beginn eines wirtschaftlichen Aufschwungs zu. Mit zunehmender Dauer des Aufschwungs setzt allerdings für Männer ein entgegengesetzter Einfluss ein und das Risiko einer Beschäftigungsbeendigung steigt aufgrund der hohen Wachstumsraten der Vorperioden.
\end{abstract}

JEL: C41, J62, J63

Olaf Hübler

Institut für empirische Wirtschaftsforschung

Leibniz Universität Hannover

Königsworther Platz 1, D-30167 Hannover

Email: huebler@ewifo.uni-hannover.de

Thomas Walter

Zentrum für Europäische Wirtschaftsforschung (ZEW)

Postfach 103443, D-68034 Mannheim

Email: walter@zew.de 


\section{Einleitung}

Die Entwicklung der Beschäftigungsstabilität ist seit einiger Zeit vermehrt Gegenstand arbeitsökonomischer Analysen. Empirische Studien, die eine Veränderung der Beschäftigungsstabilität im Zeitverlauf untersuchen, liefern allerdings keine eindeutigen Ergebnisse. Einige Untersuchungen berichten von einer mit der Zeit abnehmenden Stabilität, andere erkennen nahezu keine Veränderungen. Wiederum andere Untersuchungen ermitteln eine zunehmende Beschäftigungsstabilität. Veränderungen der Beschäftigungsstabilität müssen jedoch keinem langfristigen Trend folgen, sondern können auch konjunkturbedingten Schwankungen unterliegen. Dies ist der Fall, wenn sich Mobilitätsprozesse auf dem Arbeitsmarkt während wirtschaftlicher Auf- und Abschwünge unterscheiden. Hierzu existieren zwar einige wenige empirische Untersuchungen (Burda und Wyplosz 1994, Garloff 2004). Allerdings beruhen diese überwiegend auf aggregierten Daten. Mit solchen Daten lassen sich häufig lediglich Unterschiede in der Beschäftigungsstabilität während verschiedener konjunktureller Phasen erkennen, aber es lässt sich nicht ermitteln, ob die Unterschiede tatsächlich auf den Konjunkturverlauf oder auf andere Einflussgrößen zurückzuführen sind, die ebenfalls zyklischen Schwankungen unterliegen. Hier soll daher mit dem Sozio-ökonomischen Panel ein mikroökonomischer Datensatz eingesetzt werden, um so eine differenziertere Analyse des Einflusses konjunktureller Schwankungen auf die Stabilität von Beschäftigungsverhältnissen zu ermöglichen. Die individuelle Betriebszugehörigkeitsdauer wird dabei als zentrale Kategorie herangezogen.

Der Beitrag ist wie folgt gegliedert: In Abschnitt 2 wird ein Literaturüberblick zu vorhandenen empirischen Arbeiten über langfristige Beschäftigungsstabilität gegeben. Abschnitt 3 dient der Diskussion ökonomisch-theoretischer Überlegungen zur Erklärung der Stabilität von Beschäftigungsverhältnissen, wobei insbesondere auf einen möglichen Einfluss konjunktureller Schwankungen eingegangen wird. Abschnitt 4 enthält die eigene empirische Analyse. Nach der Beschreibung des Datensatzes wird kurz auf methodische Aspekte der Zeitdaueranalyse eingegangen, die hier in Form parametrischer Ansätze als ökonometrisches Analyseverfahren zum Einsatz kommt. Anschließend werden die mit dieser Methodik erzielten Ergebnisse präsentiert und interpretiert. Ein Fazit in Abschnitt 5 schließst den Beitrag ab. 


\section{Empirische Studien zur langfristigen Beschäftigungs- stabilität}

Durch die zunehmende Integration der Weltwirtschaft und den daraus resultierenden Anpassungsdruck für die am internationalen Wettbewerb beteiligten Volkswirtschaften sind die Arbeitsmärkte nahezu aller Industriestaaten Veränderungsprozessen unterworfen. Die Entwicklung der Beschäftigungsstabilität im Zeitverlauf ist daher nicht nur in Deutschland, sondern auch in anderen Ländern Gegenstand zahlreicher Diskussionen und hat entsprechend zu einer Vielzahl empirischer Untersuchungen geführt.

Nicht überraschend finden sich die meisten Beiträge für den Arbeitsmarkt der USA. Ein Großteil der US-amerikanischen Studien beruht entweder auf Daten des Current Population Survey (CPS) oder auf Daten der Panel Study of Income Dynamics (PSID). Nahezu alle Studien, die auf dem CPS basieren, finden für die 1980er und frühen 1990er Jahre auf gesamtwirtschaftlicher Ebene keine signifikanten Veränderungen in der Stabilität von Beschäftigungsverhältnissen. Allerdings lassen sich für einige Teilgruppen des Arbeitsmarktes Veränderungen in der Beschäftigungsstabilität erkennen. So ermittelt Farber (1995) für den Zeitraum von 1973 bis 1993 eine abnehmende Beschäftigungsdauer für geringqualifizierte Arbeitskräfte. Zu einem ähnlichen Ergebnis kommen Diebold et al. (1997). Für den weiteren Verlauf der 1990er Jahre finden Neumark et al. (1999) einen leichten Rückgang der Beschäftigungsstabilität insgesamt, wobei die Autoren aber darauf hinweisen, dass es voreilig wäre, in diesem Zusammenhang von einem langfristigen Trend zu sprechen. Die Ergebnisse, die auf der PSID basieren, sind sehr gemischt. So berichten beispielsweise Rose (1995) und Boisjoly et al. (1998) von einer starken Abnahme der Beschäftigungsstabilität. Jaeger und Stevens (1999) können hingegen kaum Veränderungen feststellen.

Auch für Großbritannien ergibt sich kein eindeutiges Bild. Burgess und Rees (1996) ermitteln für den Zeitraum von 1975 bis 1992 auf Basis des General Household Survey eine Abnahme der durchschnittlichen Betriebszugehörigkeitsdauer für Männer. Auch für Frauen treten Veränderungen auf. Allerdings folgen diese keinem langfristigen Trend. Die Autoren sehen in ihren Ergebnissen insgesamt aber keinen Hinweis auf eine zunehmende Instabilität von Beschäftigungsverhältnissen. Auch in einer stärker nach Untergruppen differenzierenden Folgestudie können Burgess und Rees (1998) keine Veränderung der Beschäftigungsstabilität zwischen den 1970er und 1990er Jahren feststellen. Gregg und Wadsworth (1995) hingegen untersuchen den gleichen Zeitraum mit Daten des British Labour Force Survey und ermitteln eine abnehmende Beschäftigungsstabilität. In einer ergänzenden Studie bestätigen Gregg und Wadsworth (2002) dieses Ergebnis für die späten 
1990er Jahre. Vor allem ältere Arbeitskräfte mit langer Betriebszugehörigkeitsdauer und Frauen mit Kindern sehen sich mit einer verringerten Stabilität ihrer Beschäftigungsverhältnisse konfrontiert. Auch Booth et al. (1999) ermitteln auf einer retrospektiv erhobenen Datenbasis des British Household Panel Survey eine Abnahme der Beschäftigungsstabilität.

Für Deutschland zeigen Bergemann und Schneider (1998) auf Basis des Sozio-ökonomischen Panels mit nichtparametrischen Methoden der Ereignisanalyse, dass die mittlere abgeschlossene Betriebszugehörigkeitsdauer von Arbeitskräften im Zeitraum von 1984 bis 1993 zurückgegangen ist. Dieses Ergebnis gilt für Männer und Frauen. Stärker ausgeprägt ist die Entwicklung jedoch für Männer. Bergemann und Schneider kommen zu dem Ergebnis, dass die Beschäftigungsstabilität nicht zwingend einem abnehmenden Trend folgen muss, sondern dass die festgestellte Entwicklung der Betriebszugehörigkeitsdauer auch auf konjunkturellen Einflüssen beruhen kann. Eine detaillierte Untersuchung dieser Vermutung unterbleibt allerdings.

Winkelmann und Zimmermann (1998) können keine Abnahme der Beschäftigungsstabilität feststellen. Vielmehr berichten sie von einer zunehmenden Stabilität. Auf Basis des Sozio-ökonomischen Panels für den Zeitraum 1974 bis 1994 ermitteln sie unter Verwendung eines Zähldatenmodells, dass die Anzahl an Arbeitsplatzwechseln für eine Arbeitskraft im Zeitraum von 1984 bis 1994 signifikant gegenüber der Zahl an Wechseln in der Periode von 1974 bis 1984 abgenommen hat. Dies wird als Hinweis auf eine zunehmende Beschäftigungsstabilität interpretiert.

Mertens (1999), Bergemann und Mertens (2001) sowie Bergemann (2004) stellen durch die Schätzung semiparametrischer Cox-Modelle eine abnehmende Beschäftigungsstabilität in Deutschland fest. Auf Basis der Daten des Sozio-ökonomischen Panels berichtet Bergemann (2004), dass der Medianwert der bisherigen Betriebszugehörigkeitsdauer für Männer von 9,4 Jahren im Jahr 1984 auf 7,5 Jahre im Jahr 1999 gesunken ist. Für Frauen findet sich für den Gesamtzeitraum keine Abnahme der mittleren bisherigen Betriebszugehörigkeitsdauer.

Eine in den 1990er Jahren kaum veränderte Beschäftigungsstabilität wird von Gerlach und Stephan (2005) sowie von Boockmann und Steffes (2005) bestätigt. In diesen Studien wird anders als in den zuvor zitierten ein Linked-Employer-Employee-Datensatz eingesetzt. Im ersten Fall ist dies die Niedersächsische Gehalts- und Lohnstrukturerhebung für die Jahre 1990, 1995 und 2001 und im zweiten Fall wird der LIAB für die Jahre 1996 bis 2001 herangezogen. Durch diese verknüpften Datensätze lassen sich sowohl Informationen über Arbeitnehmer als auch Arbeitgeber in der Analyse einbeziehen. Während Gerlach und Stephan ein Mehrebenen-Modell schätzen, nutzen Boockmann und Steffes die Zeit- 
daueranalyse und schätzen ein Cox-Modell.

Grotheer und Struck (2003) ermitteln eine im Verlauf der 1990er Jahre abnehmende Stabilität von Beschäftigungsverhältnissen. Unter Verwendung der BIBB/IAB-Erhebung und der Beschäftigtenstichprobe des Instituts für Arbeitsmarkt- und Berufsforschung (IAB) berichten die Autoren für diesen Zeitraum von einem Anstieg des Anteils kurzfristiger Beschäftigungsverhältnisse an allen Beschäftigungsverhältnissen. Zudem ermitteln sie einen sinkenden Anteil an Arbeitskräften mit mehr als zehn Jahren Betriebszugehörigkeitsdauer. Diese Ergebnisse können von Erlinghagen (2002, 2004) sowie Erlinghagen und Knuth (2004), die ebenfalls mit der IAB-Beschäftigtenstichprobe arbeiten, nicht bestätigt werden. Vielmehr wird nach diesen Ergebnissen in der zweiten Hälfte der 1980er Jahre ein Anstieg der Beschäftigungsstabilität festgestellt. Das höhere Stabilitätsniveau hat sich zumindest bis Mitte der 1990er Jahre gehalten und ist nicht durch eine gegenläufige Entwicklung revidiert worden.

Insgesamt bleibt die Entwicklung der Beschäftigungsstabilität der vergangenen Jahre in Deutschland unklar. Allerdings deuten die Ergebnisse an, dass die Beschäftigungsstabilität Veränderungen im Zeitablauf unterliegt, auch wenn die Richtung dieser Veränderung nicht eindeutig ist. Da die genannten Ergebnisse der bisherigen Studien auch auf Schwankungen der Beschäftigungsstabilität hinweisen, ist eine Berücksichtigung konjunktureller Einflüsse von besonderem Interesse. Hieran mangelt es bisher. Die vorgelegte Untersuchung will diese Lücke zumindest teilweise schließen und analysiert die Entwicklung der Beschäftigungsstabilität anhand der Betriebszugehörigkeitsdauer in Westdeutschland im Konjunkturverlauf für den Zeitraum von 1984 bis 2005.

\section{Theoretischer Hintergrund}

Die Stabilität von Beschäftigungsverhältnissen steht in engem Zusammenhang mit Mobilitätsprozessen auf dem Arbeitsmarkt. Als Mobilität sollen hier Arbeitsplatzwechsel, Wechsel aus Arbeit in Arbeitslosigkeit und komplette Rückzüge vom Arbeitsmarkt verstanden werden. Allen diesen Bewegungen ist gemeinsam, dass sie mit der Beendigung eines Beschäftigungsverhältnisses einhergehen und somit dessen Dauer beziehungsweise Stabilität beeinflussen. Winkelmann und Zimmermann (1998, S. 1414) sprechen in diesem Zusammenhang davon, dass Beschäftigungsstabilität und Arbeitskräftemobilität zwei Seiten derselben Medaille sind. Eine hohe Beschäftigungsstabilität impliziert eine geringe Mobilität, und umgekehrt folgt aus einem hohen Grad an Mobilität eine höhere Instabilität. Im Folgenden soll daher unter besonderer Beachtung konjunktureller Aspekte auf Mobilitätsprozesse eingegangen werden, um auf diese Weise Aussagen über die Entwick- 
lung der Stabilität von Beschäftigungsverhältnissen abzuleiten. Gängige ökonomische Arbeitsmarkttheorien sind daraufhin zu prüfen, ob sie einen Erklärungsbeitrag für zyklische Schwankungen der Betriebszugehörigkeitsdauer leisten.

\subsection{Arbeitsplatzsuchtheorie}

Bei der Suche on-the-job (Blanchard, Diamond, Hall und Murphy 1990) wird ein Arbeiter sein bisheriges Beschäftigungsverhältnis beenden, wenn er durch die Aufnahme einer neuen Arbeit bei einem anderen Arbeitgeber einen höheren Nutzen als gegenwärtig erzielen kann und zudem durch den höheren Nutzen auch die Kosten, die zur Suche der neuen Stelle aufgewendet wurden, kompensiert werden können. Wenn sich der Nutzen ausschließlich aus dem Lohn ergibt, kommt es immer dann zu einem Wechsel des Arbeitsplatzes, wenn die Entlohnung im neuen Beschäftigungsverhältnis höher als zuvor ist und auch die Suchkosten gedeckt werden können. Falls sich der Nutzen aus dem Lohn und zusätzlich aus nichtpekuniären Elementen wie beispielsweise den Arbeitsbedingungen zusammensetzt, muss eine differenziertere Betrachtung erfolgen. Gleiches gilt bei Berücksichtigung von Unsicherheit. Ein Arbeiter wird bei Unsicherheit erst dann wechseln, wenn er durch den Wechsel einen deutlich höheren Lohn erhält und die Suchkosten gering sind, so dass er auch dann noch einen Nutzenanstieg erwarten kann, wenn sich die nichtpekuniären Elemente des neuen Arbeitsplatzes als nachteilig im Vergleich zu den bisherigen herausstellen sollten.

Da die Suchtheorie (Mortensen 1986) auf diese Weise Aussagen über das Kündigungsverhalten von Arbeitnehmern trifft, trägt sie zur Erklärung der Stabilität von Beschäftigungsverhältnissen bei. Zugleich liefert sie Hinweise auf eine mögliche Entwicklung der Beschäftigungsstabilität im Konjunkturverlauf. In einer Aufschwungsphase ist es wahrscheinlich, dass neue attraktive Arbeitsplätze entstehen, die sich durch eine gute Entlohnung auszeichnen. Es sollten daher vermehrt Mobilitätsprozesse durch Kündigungen von Arbeitnehmern, die den Arbeitsplatz wechseln, initiiert werden. Folglich wird die Stabilität der bisher bestehenden Beschäftigungsverhältnisse negativ beeinflusst. In einem wirtschaftlichen Abschwung ist die Anzahl attraktiver Stellen hingegen begrenzt. Da in einer solchen Phase kaum Lohnerhöhungen realisiert werden können, sollte die freiwillige Mobilität der Arbeitnehmer gering sein. Die Suchtheorie impliziert somit eine prozyklische Entwicklung von Arbeitnehmerkündigungen. Im Boom sind Kündigungen zahlreicher als in der Rezession. Allerdings lassen sich auf Basis der reinen Suchtheorie keine Aussagen über die Entwicklung arbeitgeberseitiger Entlassungen treffen.

$\mathrm{Zu}$ beachten sind allerdings auch "discouraged worker"- und "added worker effects". Erstere beschreiben die Wirkungen abnehmender Attraktivität von Arbeitsplätzen aufgrund 
sinkender Löhne und die geringen Chancen, bei Entlassungen einen neuen Arbeitsplatz zu finden. Soweit keine Notwendigkeit zur Erwerbstätigkeit besteht, erfolgt eine vorübergehende Abwanderung in die Nichterwerbstätigkeit. Das wird am ehsten bei Frauen in traditionellen Familienverbänden mit einem gut verdienenden Ehemann erwartet. Bei Familien mit nur einer erwerbstätigen Person, die wenig und im wirtschaftlichen Abschwung noch weniger verdient oder gar entlassen wird, entsteht zunehmend die Notwendigkeit, dass sich auch der andere Ehepartner eine Beschäftigung sucht ("added worker effect"). In beiden Fällen, vor allem aber im letzteren Fall bewirkt dies, dass die durchschnittliche Betriebszugehörigkeitsdauer aufgrund der Änderungen in der Partizipation sinkt. Dem steht ein von Barrett und Södersten (1975) beschriebener "captive worker effect" gegenüber. Er besagt, dass in allgemein wirtschaftlichen Krisensituationen, in denen die Gefahr langer Arbeitslosigkeitsphasen besteht, verbunden mit hohen Einkommensverlusten, die Neigung zum freiwilligen Arbeitsplatzwechsel zurückgeht, zumindest wenn kein direkter Wechsel auf einen neuen Arbeitsplatz möglich ist.

\subsection{Humankapital und Beschäftigungsstabilität}

Auch die Humankapitaltheorie liefert einen Erklärungsansatz für die Stabilität von Beschäftigungsverhältnissen. Anders als die Suchtheorie jedoch beinhaltet dieser Ansatz, der auf den grundlegenden Arbeiten von Becker (1962) und Mincer (1958) beruht, so-

wohl das Verhalten der Arbeitnehmer als auch das der Arbeitgeber. Üblicherweise wird die Finanzierung des betriebsspezifischen Humankapitals von beiden Seiten gemeinsam vorgenommen. Durch Kosten- und Ertragsteilung werden die Anreize einer Kündigung seitens des Arbeitnehmers verringert. Gleichzeitig hat das Unternehmen ein geringeres Interesse daran, den Arbeiter zu entlassen. Das Beschäftigungsverhältnis zwischen Arbeitgeber und -nehmer gewinnt an Stabilität. Es ist umso stabiler, je mehr betriebsspezifisches Humankapital diesem innewohnt. Der Umfang des allgemeinen Humankapitals ist für die Stabilität eines Beschäftigungsverhältnisses von deutlich geringerer Bedeutung, da bei dessen Akkumulation die Kosten weniger geteilt werden.

Einflüsse auf die Beschäftigungsstabilität ergeben sich nach der Humankapitaltheorie vor allem über die Investitionsentscheidung bezüglich des zu akkumulierenden betriebsspezifischen Humankapitals. Inwieweit diese Investitionsentscheidung allerdings vom Konjunkturverlauf abhängt, ist fraglich. Auf den ersten Blick erscheint die Akkumulation von betriebsspezifischem Humankapital langfristig nahezu immer sowohl für den Arbeitgeber als auch den Arbeitnehmer sinnvoll zu sein, da sich dadurch die Produktivität und Entlohnung erhöht. Wenn ein Arbeitnehmer ab einem bestimmten Zeitpunkt absehen kann, dass sich das Unternehmen, in dem er arbeitet, nur noch kurzfristig am Markt behaupten 
kann und eine Schließung droht, so wird er Investitionen in betriebsspezifisches Humankapital unterlassen und stattdessen versuchen, eine neue Beschäftigung in einem anderen Unternehmen zu finden. Ein solches Szenario ist vor allem in einer Rezession zu erwarten, so dass in dieser Phase Beschäftigungsverhältnisse instabiler wären als in einem Boom, in dem Humankapitalinvestitionen besonders lohnenswert erscheinen. Unternehmen neigen in rezessiven Phasen dazu, Personal auszutauschen, um auf diesem Weg das durchschnittliche Qualifikationsniveau der Beschäftigten zu erhöhen (Burda und Wyplosz 1994).

Betriebsspezifisches Humankapital wird im Zeitverlauf durch On-the-job-Training akkumuliert. Mit zunehmender Zeit und dadurch mit steigendem Humankapital erhöht sich die Produktivität des Arbeiters im jeweiligen Unternehmen und damit verbunden seine Entlohnung, so dass sich sowohl für den Arbeitgeber als auch für den Arbeitnehmer zunehmend Vorteile einer langfristigen Zusammenarbeit ergeben. Das Risiko einer Trennung nimmt daher ab (Schasse 1991, S. 60).

\subsection{Job-Turnover und Matchingtheorie}

Weitere Erklärungen für Mobilitätsprozesse finden sich im Job- und Labour-TurnoverAnsatz, der sowohl arbeitsangebots- als auch arbeitsnachfrageseitige Elemente einbezieht. Grundlage dieses Ansatzes ist die Erkenntnis, dass zur Erfassung von Bewegungen auf dem Arbeitsmarkt eine ausschließliche Betrachtung von Bestandsgrößen unzureichend ist. Vielmehr sind auch Stromgrößen wie der Job- und Labour-Turnover in die Analyse einzubeziehen (Blanchard und Diamond 1992). Ersterer lässt sich als die Summe aller in einer Volkswirtschaft während einer bestimmten Periode entstehenden und entfallenden Arbeitsplätze definieren (Schettkat 1996, S. 17). In einem wirtschaftlichen Aufschwung werden mehr neue Arbeitsplätze geschaffen als während eines Abschwungs. Der Abbau von Arbeitsplätzen verläuft hingegen antizyklisch. In einer Rezession gehen mehr Arbeitsplätze verloren als in einem Boom. Dieses Ergebnis gibt somit Hinweise auf die Entwicklung der Beschäftigungsstabilität im Konjunkturzyklus. Labor Turnover kann als Summe aller in einer Volkswirtschaft während einer bestimmten Periode neu eingegangenen und aufgelösten Beschäftigungsverhältnisse definiert werden (Schettkat 1996, S. 15). In einem wirtschaftlichen Aufschwung werden mehr Beschäftigungsverhältnisse begonnen als während einer Rezession. Dieses Ergebnis ist nicht unerwartet, da im Boom viele neue, attraktive Arbeitsplätze entstehen, die besetzt werden müssen, wohingegen in der Rezession die Nachfrage der Unternehmen nach neuen Arbeitskräften begrenzt ist.

Bei der Frage, ob Beschäftigungsverhältnisse beendet werden, treten im Konjunkturverlauf zwei entgegengesetzt wirkende Kräfte auf, die durch arbeitgeberseitige Entlassungen oder durch arbeitnehmerseitige Kündigungen zustande kommen. Wie durch eine Vielzahl 
an Studien ermittelt wurde, weisen Entlassungen in der Regel einen antizyklischen und Kündigungen einen prozyklischen Verlauf auf (Blanchard und Diamond 1992). Dies wird in der Stromgrößenanalyse häufig wie folgt erklärt: In einer Rezession besteht verstärkt die Gefahr, dass Arbeitsplätze unprofitabel werden und daher aus Unternehmenssicht abgebaut werden müssen. In einem wirtschaftlichen Aufschwung hingegen werden die meisten Arbeitsplätze profitabel und es besteht für Arbeitgeber kaum ein Anlass, Arbeitskräfte zu entlassen. Vielmehr benötigen Unternehmen, die von der positiven konjunkturellen Entwicklung profitieren, zusätzliche Arbeitskräfte. Unternehmen werden daher im Boom vermehrt versuchen, neue Mitarbeiter einzustellen. Dadurch, dass viele freie attraktive Stellen existieren, ist es für Arbeitskräfte einfacher, eine neue Beschäftigung zu finden. Dies gilt insbesondere auch für die Arbeitskräfte, die bereits beschäftigt sind, aber nach einer neuen Stelle suchen. Es kommt vermehrt zu Kündigungen. Durch diese Kündigungen entstehen wiederum unbesetzte Arbeitsplätze, die von anderen Arbeitskräften gefüllt werden müssen. Auf diese Weise kann es zu sogenannten vacancy chains (Akerlof, Rose, Yellen, Ball und Hall 1988) kommen, die letztendlich zu einer vergleichsweise hohen Anzahl an Kündigungen in der Phase eines Booms führen (vgl. u.a. Blanchard und Diamond 1992, S. 356f.). In einer Rezession hingegen ist die Anzahl an attraktiven Stellenangeboten begrenzt, da die Unternehmen insgesamt eine geringere Nachfrage nach Arbeitskräften entwickeln. Arbeitnehmerseitige Kündigungen gehen daher zurück.

Ein für die Arbeitsmarkttheorie zentraler Ansatz, der Mobilität im Gleichgewicht erklärt, ist die Matchingtheorie (Jovanovic 1979, Mortensen und Pissarides 1994, Mortensen und Pissarides 1999, Barlevy 2002). Eine Matchingtechnologie, analog einer Produktionstechnologie, beschreibt den Zusammenhang zwischen den Inputs (individuelle Arbeitssuche und Rekrutierungsstrategie der Betriebe) und Output des Matchingprozesses, der Arbeitsanbieter mit offenen Stellen zusammenbringt. A priori ist die Matchingqualität, die zum Ausdruck bringt, wie gut das Profil eines Arbeitsanbieters mit den gewünschten Charakteristika des zu besetzenden Jobs zusammenpasst, nicht beobachtbar, sondern ein Erfahrungsgut. Die Beschäftigungsstabilität hängt von der Qualität eines Matches ab. Der Konjunkturverlauf kann die Stabilität eines Beschäftigungsverhältnisses beeinflussen, wenn er dessen zugrunde liegende Matchqualität beeinflusst. Zu beachten ist, dass in Rezessionsphasen die Anforderungen an Arbeitsplätze häufig erhöht werden und dies bedeutet, dass sich aus Sicht der Unternehmen die Matchingqualität verschlechtert. Die Arbeitgeberseite wird daher versuchen über Entlassungen und Neueinstellungen (Caballero und Hammour 1996) besser qualifizierte Arbeitskräfte zu bekommen, also ein Upgrading der Belegschaft zu ereichen (Krause und Lubik 2006). Dies sollte in einer Phase, die durch ein Überangebot an Arbeitskräften gekennzeichnet ist, auch möglich sein und führt zu einer Verbesserung der Matchqualität. In Boomphasen ergibt sich aus Sicht der 
Beschäftigten eine Verschlechterung der Matchingqualität, solange keine Mobilitätsprozesse einsetzen. Da insgesamt mehr Arbeit nachgefragt wird, sinken die Anforderungen bei Neueinstellungen. Die bereits länger Beschäftigten fühlen sich nunmehr nicht ausbildungsadäquat eingesetzt und haben daher eine zunehmende Neigung, ihren Arbeitsplatz $\mathrm{zu}$ verlassen.

Das Konzept der Matchingfunktion (Burda 1993, Mortensen und Pissarides 1999), die Ströme von Arbeitskräften auf Arbeitsplätze bei gegebenen Bestand an Arbeitslosen und offenen Stellen modelliert, also den Umfang an Stellenbesetzungen beschreibt, ist aus wirtschaftstheoretischer Sicht hier ein geeignetes Analyseinstrument. Zu beachten sind aber zumindest zwei Aspekte, die unter konjunkturellem Gesichtspunkt zu einem unterschiedlichen Matchingfunktionsverlauf in Auf- und Abschwungphasen führen (Hübler 1993, S. 201). Erstens ist zu erwarten, dass zyklische Auf- und Abwärtsbewegungen unterschiedliche Verläufe haben. Eine Bewegung aus der Krise ist anfangs mit einem stärkeren Anstieg der offenen Stellen als einer Reduktion der Arbeitslosenzahlen gekennzeichnet. Da in der Krise sich im Allgemeinen die Anforderungen an einen Arbeitsplatz erhöhen, führt ein Güternachfrageanstieg zunächst nicht zur Einstellung von Arbeitslosen, da sie die Anforderungen nicht erfüllen. Erst wenn diese gelockert werden, da die Güternachfrage nicht mehr nur durch Ausweitung der Arbeitszeit zu befriedigen ist, geht auch die Arbeitslosenzahl zurück. Verstärkt wird der Effekt eines verzögerten Abbaus der Arbeitslosigkeit dadurch, dass mit der Arbeitslosigkeit ein Verlust an Humankapital einhergeht und daher Einarbeitungskurse notwendig sind, bevor nach einer Wiedereinstellung das frühere Produktivitätsniveau erreicht wird. Im Boom verläuft die Bewegung umgekehrt. Zunächst wird bei Nachfragerückgang versucht, die Arbeitskräfte zu halten. Lediglich freie Stellen werden abgebaut. Erst bei Verschärfung des Nachfragerückgangs kommt es zu Entlassungen.

Zweitens sind Arbeitskräfte nicht, wie im Modell unterstellt, homogen. Angenommen, es gibt zwei Qualifikationsniveaus (Q1>Q2). Wenn Personen mit Q1 die Tätigkeiten von Q2-Arbeitskräften ausüben können, aber nicht umgekehrt, wenn die Unernehmen in Q1, nicht jedoch in Q2 investiert haben, dann ist es sofort einleuchtend, dass die UV-Kurven (unemployment vacancy curves) unterschiedlich verlaufen. Arbeitskräfte mit Q1 werden weniger schnell entlassen, aber u.U. für Tätigkeiten eingesetzt, die bisher Q2-Arbeitskräfte ausgeübt haben, da generell die Anforderungen an einen Arbeitsplatz erhöht werden. In diesem Fall kann es in einer Krise durchaus noch freie Stellen für frühere Q1-Arbeitsplätze geben, während dies für frühere Q2-Arbeitsplätze weniger zu erwarten ist. 


\section{Empirische Analyse}

\subsection{Datensatz und Variablen}

Als Datenbasis für die empirische Untersuchung wird das Sozio-ökonomische Panel (SOEP) für den Zeitraum von 1984 bis 2005 verwendet. Das SOEP ist eine repräsentative Wiederholungsbefragung von Personen in Privathaushalten und besteht unter anderem aus einem Kalendarium. Dort werden von den befragten Personen in jeder Erhebungswelle detaillierte monatliche Angaben zu ihrer Tätigkeit während des jeweiligen Vorjahres erfasst. ${ }^{1}$ Mittels dieser Angaben wird ein Spelldatensatz generiert, in dem jede Beobachtung eine Tätigkeitsperiode wiedergibt und somit den Zeitraum von der Aufnahme bis zur Aufgabe der Tätigkeit umfasst. Dabei wird die Betrachtung auf Spells der Voll- und Teilzeiterwerbstätigkeit begrenzt. Für jeden Spell ist der Beginn und das Ende durch die Angabe des jeweiligen Jahres und des Monats festgehalten. Es ist jedoch anzumerken, dass die im Spelldatensatz erfassten Erwerbstätigkeitsperioden ohne weitere Korrekturen nicht mit Beschäftigungsverhältnissen gleichgesetzt werden können, da eine Erwerbstätigkeitsperiode bei mehr als einem Arbeitgeber verrichtet werden kann. Wenn eine Befragungsperson ihren Arbeitsplatz wechselt, ohne dass eine Erwerbsunterbrechung eintritt, so ist im Kalendarium lediglich ein Spell der Erwerbstätigkeit erfasst, obwohl zwei Beschäftigungsverhältnisse vorliegen. Aus diesem Grund werden für jede Befragungsperson aus den übrigen Angaben im SOEP zusätzliche Informationen hinsichtlich der Aufnahme und Beendigung von Beschäftigungsverhältnissen eingeholt. ${ }^{2}$ Mit diesen Zusatzinformationen werden Erwerbstätigkeitsperioden bei mehr als einem Arbeitgeber aufgespalten, so dass sie nunmehr

\footnotetext{
${ }^{1}$ Es ist allgemein bekannt, dass retrospektiv erhobene Daten fehlerbehaftet sind. Dies gilt auch für das SOEP, wie die Untersuchungen von Jürges (2005) zeigen. So werden Arbeitslosigkeitperioden tendenziell unterschätzt oder es wird bisweilen gar nicht über sie berichtet. Ebenso sind Fehler bei der Angabe der Betriebszugehörigkeitsdauer zu erwarten. Für die Schätzung ist das Problem von Fehlern in den Variablen allerdings meist weniger gravierend, wenn es sich um Fehler in der endogenen Variablen wie im vorliegenden Fall handelt - vgl. Hübler 1989, S.224.

${ }^{2}$ Insbesondere wird hier Gebrauch von Antworten auf die Fragen: "Wann haben Sie bei Ihrer letzten Stelle aufgehört zu arbeiten?" und "Wann haben Sie Ihre jetzige Stelle angetreten bzw. die Arbeit aufgenommen?" gemacht. Mit den Angaben der befragten Personen zu diesen beiden Fragen in aufeinanderfolgenden Befragungswellen lassen sich je nach Befragungszeitpunkt für ein Kalenderjahr bis zu zwei Beschäftigungswechsel identifizieren. Vielfach ist jedoch nur ein Wechsel trennscharf beobachtbar. Wie allerdings ein Abgleich mit dem Regionalfile der IAB-Beschäftigtenstichprobe für den Zeitraum von 1984 bis 2001 zeigt, treten mehrmalige unterjährige Beschäftigungswechsel vergleichsweise selten auf. Mehr als $85 \%$ der Personen, die unterjährig wechseln, bleiben innerhalb eines Kalenderjahres in diesem neuen Beschäftigungsverhältnis und wechseln nicht erneut. Beschäftigungswechsel sind somit durch das SOEP hinreichend erfasst, auch wenn letztlich eine Unterrepräsentativität des SOEP bezüglich kurz andauernder Beschäftigungsverhältnisse nicht mit Sicherheit ausgeschlossen werden kann.
} 
deutlich besser mit den jeweiligen Beschäftigungsverhältnissen übereinstimmen.

Auf diese Weise entsteht aus dem Spelldatensatz, der zunächst Erwerbstätigkeitsperioden erfasste, ein Spelldatensatz, der für jede Befragungsperson die meisten Beschäftigungsverhältnisse enthält, die mit einer Voll- oder Teilzeiterwerbstätigkeit verbunden sind. Jeder Beobachtungswert in diesem neuen Spelldatensatz gibt folglich ein Beschäftigungsverhältnis wieder. Zu beachten ist, dass zensierte und abgeschnittene Beschäftigungsverhältnisse auftreten können. Dies verlangt eine besondere Berücksichtigung bei der Schätzung - vgl. Abschnitt 4.2. In dem hier verwendeten Datensatz besteht vor allem das Problem der Rechtszensierung von Beschäftigungsverhältnissen. ${ }^{3}$ Ein rechtszensiertes Beschäftigungsverhältnis liegt vor, wenn ein Beschäftigungsverhältnis zu dem Zeitpunkt, an dem es zuletzt beobachtet wurde, noch andauert. In diesem Fall lässt sich lediglich die Wahrscheinlichkeit ermitteln, dass bis zum Zeitpunkt der Zensierung noch kein Ereignis eingetreten ist, das zur Beschäftigungsbeendigung geführt hat. Abgeschnittene Beschäftigungsverhältnisse sind dadurch gekennzeichnet, dass für sie für einen gewissen Zeitraum keine Beobachtungen vorliegen, aber a posteriori bekannt ist, dass während dieser Zeit kein Ereignis eingetreten ist, das zu einer Beschäftigungsbeendigung geführt hat. ${ }^{4}$ Für jedes Beschäftigungsverhältnis ist dessen Dauer erfasst.

Insgesamt ist die Beschäftigungsdauer in dieser Untersuchung von zentraler Bedeutung, da sie als Maß der Stabilität eines Beschäftigungsverhältnisses dient. Im Folgenden wird daher die Dauer eines Beschäftigungsverhältnisses als zu erklärende Variable verwendet. Zur Bildung erklärender Variablen werden aus dem SOEP individuelle Merkmale der Beschäftigten (u.a. Alter, Schulbildung, berufliche Stellung) sowie betriebliche Charakteristika (u.a. Firmengröße, Wirtschaftssektor) erhoben.

Die gesamte Untersuchung beruht auf folgender Einschränkung des Datensatzes: Es werden nur Personen betrachtet, die in Westdeutschland ${ }^{5}$ leben und im Alter von 16 bis 65 Jahren sind. Auszubildende, Praktikanten, Freiberufler, Selbstständige und Beamte werden von der Analyse ebenso ausgeschlossen wie Beschäftigte in der Land- und Forstwirtschaft sowie der Fischerei und Fischzucht. Auch Angestellte in Privathaushalten und Immigranten werden nicht berücksichtigt. Darüber hinaus werden Personen ohne genaue Angabe zur Art ihres Schulabschlusses und solche mit einem Abschluss, der nicht den

\footnotetext{
${ }^{3}$ Linkszensierte Beschäftigungsverhältnisse, bei denen der Anfangszeitpunkt unbekannt ist, sind durch die Konstruktion des Datensatzes ausgeschlossen. Es werden nur Beschäftigungsverhältnisse berücksichtigt, die 1984 oder später angefangen haben.

${ }^{4}$ Fälle dieser Art liegen z.B. vor, wenn bekannt ist, dass ein Beschäftigungsverhältnis j zum Zeitpunkt $t_{j ; 0}$ beginnt und zum Zeitpunkt $t_{j ; 2}$ endet, aber erst ab $t_{j ; 1}>t_{j ; 0}$ beobachtet wird, da der Beobachtungsträger i im Intervall $\left[t_{j ; 0} ; t_{j ; 1}\right]$ nicht geantwortet hat, der Beginn der Beschäftigungsperiode $\left(t_{j ; 0}\right)$ jedoch retrospektiv erhoben wird.

${ }^{5}$ Westdeutschland ist hier definiert als der Gebietsstand der Bundesrepublik Deutschland vor der Wiedervereinigung im Jahr 1990 einschließlich Westberlin.
} 
üblichen deutschen Abschlüssen entspricht, aus dem Datensatz entfernt. Dies betrifft vor allem Ausländer. Ferner werden Personen, die keine Angaben zu ihrer gegenwärtigen beruflichen Stellung oder zu ihrem gegenwärtigen Arbeitgeber hinsichtlich Unternehmensgröße und Wirtschaftssektor machen, vernachlässigt. Zudem wird die Untersuchung auf den Zeitraum von 1984 bis 2005 beschränkt.

Mit dieser Abgrenzung des Datensatzes lassen sich insgesamt 8428 Beschäftigungsverhältnisse beobachten. Diese werden von 2116 Männern und 2397 Frauen eingegangen. Für Männer sind im Beobachtungszeitraum von 1984 bis 2005 insgesamt 4006 Beschäftigungsverhältnisse erfasst. Die durchschnittliche Dauer dieser Beschäftigungsverhältnisse beträgt 37,23 Monate bei einer Standardabweichung von s=45,16 Monaten. Für Frauen liegen 4422 Beschäftigungsverhältnisse mit einer Durchschnittsdauer von 33,56 Monaten vor $(s=39,72)$. Weitere deskriptive Statistiken sind in den Tabellen 1a und 1b dargestellt. Ziel unserer Untersuchung ist es, den Einfluss des Konjunkturzyklus auf die Stabilität von Beschäftigungsverhältnissen zu bestimmen. Da im SOEP keine Angaben zur konjunkturellen Entwicklung während des Beobachtungszeitraums enthalten sind, müssen entsprechende Indikatoren aus externer Quelle herangezogen werden. Hier wird auf Erhebungen des Arbeitskreises "Volkswirtschaftliche Gesamtrechnung der Länder" (2007) zurückgegriffen. Zur Verfügung stehen auf Jahresbasis ermittelte Wachstumsraten des Bruttoinlandsprodukts für den Zeitraum von 1970 bis 2006 für jedes deutsche Bundesland. Mit diesen regional und zeitlich differenzierten Wachstumsraten, die auf konstanten Preisen von 2000 beruhen, ist eine hinreichende Erfassung des Konjunkturverlaufs möglich. ${ }^{6}$ Auf Basis der verfügbaren Wachstumsraten werden zwei neue Variablen gebildet, der Durchschnittswert der aktuellen und der um ein Jahr verzögerten Wachstumsrate sowie der Durchschnittswert der um zwei und um drei Jahre verzögerten Wachstumsraten. Mit diesen beiden Variablen soll der Konjunkturverlauf wiedergegeben werden, so dass dessen Einfluss auf die Beschäftigungsstabilität bestimmt werden kann, wobei auch mögliche verzögerte Wirkungen berücksichtigt werden können. ${ }^{7}$ Abbildung 1 zeigt, dass der Durchschnittswert aus der aktuellen und der um ein Jahr verzögerten Wachstumsrate den konjunktuellen Verlauf über alle Bundesländer gut abbildet. Dort wird der aus der genannten Größe gebildete gesamtwirtschaftliche Jahresdurchschnitt verglichen mit

\footnotetext{
${ }^{6}$ Über die Messung der Konjunktur besteht in der Literatur keine einheitliche Auffassung. Neben der Arbeitslosenquote (vgl. z.B. Shimer 2005) werden aggregierte Outputgrößen wie das Bruttoinlandsprodukt oder die industrielle Nettoproduktion herangezogen, um dann führende, gleichlaufende oder nachlaufende Indikatoren zu bilden (Winker 2007). In der Praxis werden dann prozentuale Veränderungsraten gegenüber der Vorperiode gebildet oder es wird eine prozentuale Abweichung von einem langfristigen Trend bestimmt (Nierhaus 1999, S.11ff.). Für die Trendermittlung gibt es verschiedene Ansätze, so z.B. das Hodrick-Prescott-Verfahren - vgl. Shimer (2005), Winker (2007).

${ }^{7}$ Für ein entsprechendes Vorgehen vgl. Bergemann (2004, S. 33).
} 
der trendbereinigten gesamtwirtschaftlichen Wachstumsrate des BIP pro Jahr, wobei der Trend nach dem Hodrick-Prescott(HP)-Verfahren berechnet ist.

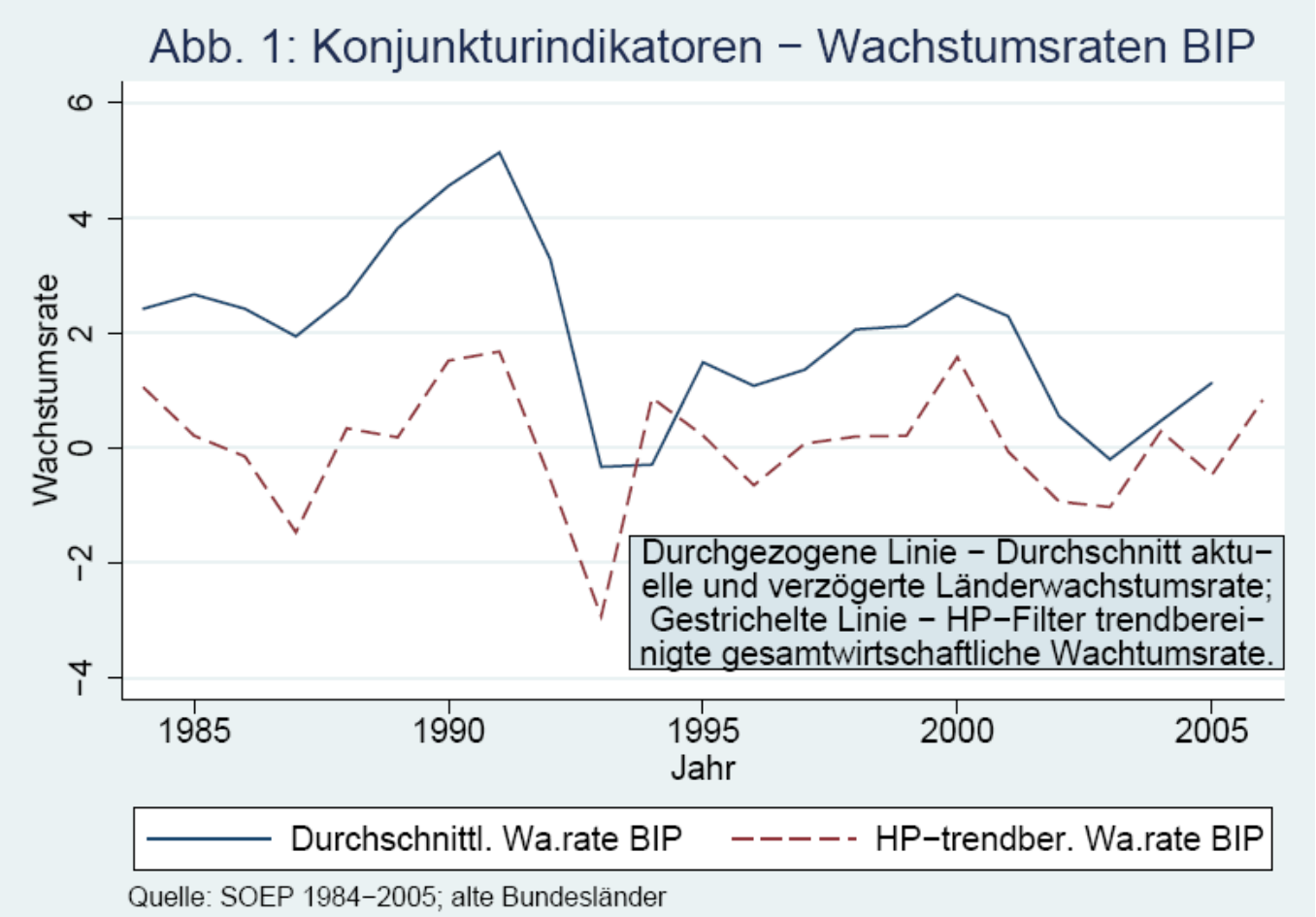

\subsection{Methodisches Vorgehen}

Um die Entwicklung der Beschäftigungsstabilität im Konjunkturverlauf empirisch zu untersuchen, soll auf parametrische Methoden der Ereignisanalyse zurückgegriffen werden. Mit der Weibull-Verteilung und der log-logistischen Verteilung kommen zwei sehr häufig benutzte Modelle zur Anwendung. Der zeitliche Verlauf der Hazardrate im Weibullmodell ist abhängig von einem Parameter $\gamma$. Wenn $\gamma$ kleiner als Eins ist, fällt die Hazardrate bei zunehmendem $t$ monoton. Für $\gamma=1$ ist die Hazardrate konstant und für $\gamma>1$ ist sie monoton steigend (vgl. Lee und Wang 2003, S. 139f.).

Auch der Verlauf der Hazardrate der log-logistischen Verteilung ist abhängig von einem Parameter $(\delta)$. Für $\delta>1$ steigt die Hazardrate zunächst an, bis sie ihr Maximum erreicht. Anschließend nimmt sie monoton ab. Die Hazardrate hat in diesem Fall einen nichtmonotonen, umgekehrt u-förmigen Verlauf. Falls $\delta \leq 1$ gilt, ist die Hazardrate monoton fallend.

Die Humankapitaltheorie kann ein solch monoton fallendes Risiko erklären - vgl. Abschnitt 3.2. Für einen nichtmonotonen, umgekehrt u-förmigen Verlauf der Hazardrate lassen sich hingegen Argumente aus der Matchingtheorie ableiten. Wird angenommen, dass ein Beschäftigungsverhältnis den Charakter eines Erfahrungsgutes besitzt, so wird 
das Risiko einer Beschäftigungsbeendigung zunächst ansteigen und später fallen. Zu Beginn des Beschäftigungsverhältnisses müssen Erfahrungswerte hinsichtlich der Qualität des Matches zwischen Arbeitgeber und Arbeitnehmer gesammelt werden. Erst wenn diese Werte vorliegen, kann eine Entscheidung über die Fortführung oder Beendigung des Matches getroffen werden. Matches mit schlechter Qualität werden beendet, gute Matches werden fortgesetzt. Da somit nur gute Matches langfristig andauern, steigt das Risiko einer Beschäftigungsbeendigung anfangs, nimmt nach einer gewissen Zeit aber wieder ab, so dass ein umgekehrt u-förmiger Verlauf der Hazardrate resultiert (vgl. Jovanovic 1979, S. 980f.).

Die Einbeziehung erklärender Variablen in die Schätzfunktion kann auf unterschiedliche Art vorgenommen werden. Hier erfolgt die Erfassung des Einflusses der erklärenden Variablen auf Basis der Accelerated Failure Time (AFT) Modelle. Die Grundform eines AFT-Modells lässt sich durch die Gleichung

$$
\ln T_{j}=\beta_{0}+\boldsymbol{x}_{j}^{\prime} \boldsymbol{\beta}_{x}+\omega \cdot \varepsilon_{j},
$$

darstellen, wobei $\ln T_{j}$ die logarithmierte Zeitdauer des Beschäftigungsverhältnisses $j$ symbolisiert und der Vektor $\boldsymbol{x}_{j}^{\prime}=\left(x_{1 j}, \ldots, x_{k j}\right)$ die Werte von $k$ erklärenden Variablen, die für dieses Beschäftigungsverhältnis erhoben wurden, beinhaltet. Der Einfluss der Variablen auf die logarithmierte Zeitdauer des Beschäftigungsverhältnisses wird durch den Vektor $\boldsymbol{\beta}_{x}^{\prime}=\left(\beta_{1}, \ldots, \beta_{k}\right)$ wiedergegeben. $\beta_{0}$ ist ein für alle Beschäftigungsverhältnisse konstanter Einflussfaktor, $\omega$ ist ein Skalierungsparameter und $\varepsilon_{j}$ stellt einen Störterm dar. Für die Verteilung der Zeitdauer $T_{j}$ lassen sich verschiedene Verteilungen unterstellen, so z.B. die Weibull- und die log-logistische Verteilung.

Werden nicht nur unzensierte Beschäftigungsverhältnisse beobachtet, sondern treten auch zensierte und abgeschnittene Beschäftigungsverhältnisse auf, so ist die Likelihood-Funktion gegenüber dem Standardfall zu modifizieren (Cleves et al. 2004, S.35f). Üblicherweise existieren auch unbeobachtbare Einflussgrößen, die die Beschäftigungsdauer mitbestimmen. Diese unbeobachtbaren Größen können sowohl auf Seiten der Arbeitnehmer als auch auf Seiten der Arbeitgeber bestehen. Zu den arbeitnehmerseitigen unbeobachtbaren Einflussgrößen auf die Beschäftigungsdauer kann beispielsweise das Verhalten einer Arbeitskraft am Arbeitsplatz oder der Umgang mit Kollegen und insbesondere Vorgesetzten gehören. Aber auch die individuelle Präferenz einer Arbeitskraft für kürzere oder längere Beschäftigungsverhältnisse muss zu den unbeobachtbaren Einflussgrößen gezählt werden.

Um unbeobachtbare Heterogenität im Modell berücksichtigen zu können, wird angenommen, dass sich die Heterogenität in einem individuenspezifischen Effekt $\nu_{i}$ ausdrückt. Dabei ist $\nu_{i}$ die Realisation einer positiven Zufallsvariablen mit einem bekannten Erwartungswert von Eins und unbekannter, zu schätzender Varianz $\psi$. Es wird unterstellt, dass $\nu_{i}$ multiplikativ auf die Hazardrate einwirkt - vgl. Cameron und Trivedi (2005, S. 613). 
Der Likelihood-Beitrag von Individuum $i$ ist abhängig vom unbekannten Heterogenitätseffekt $\nu_{i}$. Da $\nu_{i}$ selbst aber nicht geschätzt werden soll, sondern das Interesse lediglich auf der Schätzung des Vektors $\boldsymbol{\beta}$ und des Verteilungsparameters $\theta$ unter Berücksichtigung der Heterogenität beruht, muss $\nu_{i}$ eliminiert werden, um eine von $\nu_{i}$ unabhängige LikelihoodFunktion spezifizieren zu können. Dies erfordert die Wahl einer Dichtefunktion $g\left(\nu_{i}\right)$ für $\nu_{i}$. Hier wird in dem Likelihood-Beitrag $L_{i}$

$$
L_{i}\left\{\boldsymbol{\beta}, \theta, \psi \mid t_{i j}, \boldsymbol{x}_{i j}\right\}=\int_{0}^{\infty} \nu_{i}^{D_{i}} \prod_{j=1}^{J}\left\{\frac{S\left(t_{i j} \mid \boldsymbol{x}_{i j}, \boldsymbol{\beta}, \theta\right)}{S\left(t_{0 i j} \mid \boldsymbol{x}_{i j}, \boldsymbol{\beta}, \theta\right)}\right\}^{\nu_{i}}\left\{h\left(t_{i j} \mid \boldsymbol{x}_{i j}, \boldsymbol{\beta}, \theta\right)\right\}^{d_{i j}} g\left(\nu_{i}\right) d \nu_{i} .
$$

für $\nu_{i}$ eine Gammaverteilung $(\Gamma(\cdot))$ mit Erwartungswert Eins und einer Varianz $\psi$ angenommen. Mit $S(\cdot)$ wird die Survivorfunktion bezeichnet, $h(\cdot)$ entspricht der Hazardrate. Der Parameter $\theta$ steht für den jeweiligen Verteilungsparameter des unterstellten Modells. $D_{i}$ ist die Anzahl der für Individuum i erfassten unzensierten Beschäftigungsverhältnisse. Die Dummy-Variable $d_{i j}$ nimmt den Wert Eins an, wenn für $i$ ein unzensiertes Beschäftigungsverhältnis j vorliegt. Bei Rechtszensierung gilt entsprechend $d_{i j}=0$. Die Dichtefunktion $g\left(\nu_{i}\right)$ lautet

$$
g\left(\nu_{i}\right)=\frac{\nu_{i}^{(1 / \psi)-1} \exp \left\{-\nu_{i} / \psi\right\}}{\Gamma(1 / \psi) \psi^{1 / \psi}}
$$

Aus Gleichung (2) folgt für diesen Fall als Beitrag zur Log-Likelihood-Funktion

$$
\begin{aligned}
\ln L_{i}\left\{\boldsymbol{\beta}, \theta, \psi \mid t_{i j}, \boldsymbol{x}_{i j}\right\}= & \sum_{j=1}^{J} d_{i j} \ln h\left(t_{i j} \mid \boldsymbol{x}_{i j}, \boldsymbol{\beta}, \theta\right) \\
& -\left((1 / \psi)+D_{i}\right) \cdot \ln \left\{1-\psi \sum_{j=1}^{J} \ln \frac{S\left(t_{i j} \mid \boldsymbol{x}_{i j}, \boldsymbol{\beta}, \theta\right)}{S\left(t_{0 i j} \mid \boldsymbol{x}_{i j}, \boldsymbol{\beta}, \theta\right)}\right\} \\
& +D_{i} \ln \psi+\ln \Gamma\left((1 / \psi)+D_{i}\right)-\ln \Gamma(1 / \psi)
\end{aligned}
$$

(Gutierrez 2002, S. 34). Aus der Summe der Log-Likelihood-Beiträge aller Individuen ist die Log-Likelihood-Funktion der gesamten Stichprobe unter Berücksichtigung unbeobachteter Heterogenität zu bilden, die gemeinsam in Bezug auf den Vektor $\boldsymbol{\beta}$, den Parameter $\theta$ und die Varianz $\psi$ zu maximieren ist. ${ }^{8}$

\footnotetext{
${ }^{8}$ Erwähnt werden sollte, dass das hier als zeitstetig formulierte Modell bei Verwendung von Monatsdaten wie bei der vorliegenden empirischen Untersuchung nur approximativ Gültigkeit besitzt. Wooldridge (2003, S.706) schreibt jedoch: "When the measurements are fairly precise, it is sensible to treat the durations as continuous random variables“. Da Beschäftigungsverhältnisse üblicherweise am Monatsende aufhören, kann davon ausgegangen werden, dass die abgeschlossene Betriebszugehörigkeitsdauer hinreichend präzise erfasst wird. Die Annahme einer kontinuierlichen Zeitdauer entspricht durchaus üblicher Praxis - vgl. z.B. Gottschalk und Moffitt (1999) -, zumal Allison (1982) argumentiert, dass zeitstetige Modelle stets zeitdiskreten Modellen vorzuziehen sind. Es gibt aber durchaus Untersuchungen, die von einer diskreten Zeitdauer ausgehen, so z.B. Hujer, Maurer und Wellner (1999).
} 
Ein letztes methodisches Problem ergibt sich dadurch, dass aggregierte erklärende Variablen in die Schätzung eingehen. Dies führt bei Anwendung der üblichen Methoden zu verzerrten Schätzungen der Standardfehler (Moulton 1986, 1990) und kann durch Berechnung von clusterrobusten Kovarianzmatrizen korrigiert werden (Cameron und Trivedi 2005, S.837, Pepper 2002). Dieser Lösungsvorschlag bezieht sich auf lineare Modelle. Clusteringkorrektur im nichtlinearen Modell, das hier vorliegt, ist zwar bisher in der ökonometrischen Literatur kaum Aufmerksamkeit geschenkt worden (Cameron und Trivedi 2005, S. 841). Eine clusterrobuste Schätzung bietet sich jedoch auch in diesem Fall an.

\subsection{Ergebnisse}

Alle im Folgenden vorgestellten Ergebnisse beruhen auf Schätzungen des in Gleichung (1) beschriebenen AFT-Modells. Von zentralem Interesse ist der Einfluss der beiden Konjunkturvariablen auf die Dauer und damit die Stabilität eines Beschäftigungsverhältnisses. Zur Vereinfachung der Beschreibung soll im Folgenden der Durchschnittswert der aktuellen und der um ein Jahr verzögerten Wachstumsrate als erste Wachstumsrate bezeichnet werden. Der Durchschnittswert der um zwei und um drei Jahre verzögerten Wachstumsraten soll entsprechend zweite Wachstumsrate genannt werden. Die Untersuchung erfolgt getrennt für Frauen und Männer. Für beide Geschlechter wird auf Basis des gesamten Datensatzes sowohl das Weibull- als auch das log-logistische AFT-Modell geschätzt, da a priori nicht bestimmt werden kann, ob das Risiko einer Beschäftigungsbeendigung im Zeitverlauf monoton fallend ist oder anfangs steigt und dann fällt. Die wesentlichen Ergebnisse sind in Tabelle 2 zusammengefasst. Für Frauen ergeben sich unabhängig vom zugrunde liegenden Modell jeweils positive Koeffizienten für die erste und zweite Wachstumsrate. Allerdings sind diese Koeffizienten in nahezu allen Fällen statistisch insignifikant. Lediglich im log-logistischen AFT-Modell lässt sich ein leicht signifikanter Einfluss der ersten Wachstumsrate feststellen. Für Männer hat der Koeffizient der ersten Wachstumsrate sowohl im Weibull- als auch im log-logistischen AFT-Modell ein positives Vorzeichen. Für die zweite Wachstumsrate ergibt sich hingegen in beiden Modellen ein negativer Einfluss. Allerdings zeigen die Modelle keine Übereinstimmung hinsichtlich der Signifikanz der Koeffizienten. Während im log-logistischen AFT-Modell beide Koeffizienten insignifikant sind, ist der negative Einfluss der zweiten Wachstumsraten im Weibull-AFT-Modell statistisch gesichert.

Keine einheitliche Aussage liefern die beiden Modelle zudem in Bezug auf die Zeitabhängigkeit der Hazardrate. Die Schätzungen für $\gamma$ und $\delta^{-1}$ in Tabelle 2 lassen für die Gruppe der Männer eine langfristig fallende Hazardrate erkennen, allerdings zeigt das Weibull AFT Modell eine monoton fallende Hazardrate an, wohingegen das log-logistische AFT 
Modell einen nichtmonotonen Verlauf der Hazardrate ermittelt. Für die Gruppe der Frauen zeigt das log-logistische AFT Modell ebenfalls einen umgekehrt u-förmigen Verlauf der Hazardrate an. Das Weibull AFT Modell ermittelt hier einen konstanten Verlauf.

Um ein Modell präferieren zu können, wird untersucht, wie robust die Modellspezifikationen gegenüber Veränderungen des zugrunde liegenden Datensatzes sind. Dazu wird der Datensatz auf Beschäftigungsverhältnisse eingegrenzt, die länger als sechs Monate bestehen. Mit dem auf diese Weise erzeugten neuen Datensatz werden die Schätzungen der Weibull- und log-logistischen AFT-Modelle für Frauen und Männer wiederholt. Dabei ergibt sich, dass die log-logistischen AFT-Modelle weiterhin eine umgekehrt u-förmige Hazardrate anzeigen. Die Schätzwerte für den Parameter $\gamma$ im Weibull-AFT-Modell sind hingegen nicht robust. Wie Tabelle 3 zeigt, ergeben sich im log-logistischen AFT-Modell sowohl für Frauen als auch für Männer Schätzwerte für den Kehrwert des Parameters $\delta$, die signifikant kleiner als Eins sind und somit einen nichtmonotonen Verlauf der Hazardrate implizieren. Im Weibull-AFT-Modell für Männer ergibt sich $\hat{\gamma}=1,0204$. Dieser Wert ist nicht signifikant kleiner oder größer als Eins, was auf eine im Zeitverlauf konstante Hazardrate hinweist. Für Frauen folgt $\hat{\gamma}=1,1507$. Dieser Wert ist signifikant größer als Eins und deutet auf eine monoton ansteigende Hazardrate hin. Die Ergebnisse der Weibull-AFT-Modelle stimmen nicht mit den theoretischen Erwartungen überein. Sie lassen sich auch durch die veränderte Datenbasis nicht erklären. Trotz der Eingrenzung des Datensatzes ist auch weiterhin eine langfristig fallende Hazardrate zu erwarten, da noch relativ viele Beschäftigungsverhältnisse mit einer kurzen Dauer ( $<1$ Jahr) in der Untersuchung verbleiben (1402 von 7561 Beschäftigungsverhältnissen). Es wird daher das log-logistische AFT-Modell bevorzugt.

Die Schätzergebnisse für die Koeffizienten der Kontrollvariablen finden sich im Anhang. Auf eine explizite Erfassung des Lohnes als Determinante der Betriebszugehörigkeitsdauer wurde wegen möglicher gegenseitiger Abhängigkeit verzichtet. Vielmehr sind einkommensbestimmende Größen aufgenommen worden. Es liegt also eine reduzierte Form vor. In Bezug auf den Einfluss des Alters lässt sich folgendes feststellen: Bei den Männern zeigt sich für die Gruppe der 16- bis 25-Jährigen ein signifikant höheres Risiko einer Beschäftigungsbeendigung als für die Basisgruppe der 26- bis 35-Jährigen. Dieses Ergebnis lässt sich unter anderem durch sogenanntes job shopping erklären. Jüngere Arbeitnehmer wechseln häufiger ihren Arbeitsplatz, da sie zu Beginn des Berufslebens ihre individuellen Fähigkeiten durch das Ausüben verschiedener Tätigkeiten kennen lernen wollen. Ziel dieses Verhaltens ist es, schließlich den Arbeitsplatz zu finden, auf dem die Fähigkeiten nutzenmaximal eingesetzt werden können. Zudem ergibt sich für die Gruppe der 56- bis 65-jährigen Männer ein im Vergleich zur Basisgruppe erhöhtes Risiko einer Beschäftigungsbeendigung, was 
vor allem auf den Übergang vom Erwerbsleben in den Ruhestand zurückzuführen ist. Für Frauen lässt sich zwischen der Gruppe der 16- bis 25-Jährigen und der Basisgruppe der 26bis 35-Jährigen kein signifikanter Unterschied in der Beschäftigungsstabilität feststellen. Zwar ist auch bei jungen Frauen von einer erhöhten Mobilitätsneigung aufgrund des job shoppings auszugehen, allerdings befinden sich in der Basisgruppe vermehrt die Frauen, die durch Heirat oder durch Mutterschaft ihre Erwerbstätigkeit beenden oder sich zumindest zeitweise vom Arbeitsmarkt zurückziehen. Für beschäftigte Frauen im Alter von 36 bis 55 Jahren ergibt sich daher ein signifikant geringeres Risiko einer Beschäftigungsbeendigung. Anders als bei Männern lässt sich bei Frauen für die älteste Gruppe kein Unterschied in der Beschäftigungsstabilität feststellen. Dies kann einen entsprechenden Grund wie bei der jüngsten Alterskohorte haben.

Unterschiede in der Schulbildung haben sowohl bei Männern als auch bei Frauen kaum Einfluss auf die Stabilität von Beschäftigungsverhältnissen. Nachschulische Entwicklungen sind von größerer Bedeutung. So ergeben sich Unterschiede in der Beschäftigungsstabilität je nach der beruflichen Stellung. Insbesondere ungelernte und angelernte Arbeiter und Arbeiterinnen unterliegen einem erhöhten Risiko einer Beschäftigungsbeendigung. Die Kontrollgruppe besteht hier aus den Angestellten mit qualifizierter Tätigkeit. Dieses Ergebnis lässt sich dadurch erklären, dass Ungelernte und Angelernte einen vergleichsweise wenig in betriebsspezifisches Humankapital akkumuliert haben. Die Beschäftigungsstabilität ist umso höher, je mehr betriebsspezifisches Humankapital einem Beschäftigungsverhältnis innewohnt. Auch der Erwerbsstatus hat Einfluss auf die Beschäftigungsstabilität. Für Teilzeitbeschäftigte ist die Beschäftigungsstabilität geringer als für vollzeiterwerbstätige Arbeitskräfte.

Des Weiteren lässt sich für Männer eine Abhängigkeit der Beschäftigungsstabilität von der Firmengröße feststellen. In Klein- und Großbetrieben besteht ein höheres Risiko einer Beschäftigungsbeendigung als in Betrieben mit mittlerer Größe.

Eingehender ist die Entwicklung der Beschäftigungsstabilität im Konjunkturzyklus zu untersuchen. Im log-logistischen AFT-Modell ergibt sich für Frauen ein leicht signifikant positiver Einfluss der ersten Wachstumsrate auf die Beschäftigungsdauer. Der Koeffizient der zweiten Wachstumsrate ist insignifikant. Für Männer ergibt sich ebenfalls ein leicht signifikant positiver Einfluss der ersten Wachstumsrate. Zudem zeigt sich hier aber auch ein signifikant negativer Einfluss der zweiten Wachstumsrate auf die Beschäftigungsdauer. Der positive Koeffizient der ersten Wachstumsrate drückt aus, dass für Frauen und Männer die Beschäftigungsstabilität zum heutigen Zeitpunkt umso höher ist, je stärker das durchschnittliche Wirtschaftswachstum des jetzigen und des vergangenen Jahres ist. Anders ausgedrückt, kann gesagt werden, dass das Risiko einer Beschäftigungsbeendigung abnimmt, wenn die aktuelle wirtschaftliche Entwicklung günstig ist, wobei sich aktu- 
ell auf das gegenwärtige und das vergangene Jahr bezieht. Der negative Koeffizient der zweiten Wachstumsrate in der Schätzung für Männer zeigt hingegen an, dass das Risiko einer Beschäftigungsbeendigung in dieser Gruppe zum heutigen Zeitpunkt umso höher ist, je höher das Durchschnittswachstum der Wirtschaft im vorletzten und vorvorletzten Jahr war. Somit ergeben sich also für Männer bei lang anhaltenden Aufschwungsoder Abschwungsphasen zwei entgegengesetzt wirkende Einflüsse auf die Stabilität von Beschäftigungsverhältnissen. Diese entgegengesetzten Einflüsse können wie folgt beispielhaft dargestellt werden. Es sei angenommen, dass sich die Wirtschaft in einem Aufschwung befindet und dass die Wachstumsraten über mehrere Jahre vergleichsweise hoch sind. Zu Beginn des Aufschwungs wird dann die Stabilität von Beschäftigungsverhältnissen auch vergleichsweise hoch sein. Mit der Zeit verringert sich allerdings die Beschäftigungsstabilität, da das hohe Wachstum der Vorperioden das Risiko einer Beschäftigungsbeendigung erhöht. Wird hingegen angenommen, dass sich die Wirtschaft in einer Rezession befindet und die Wachstumsraten über längere Zeit gering oder gar negativ sind, so wird die Beschäftigungsstabilität anfangs zunächst abnehmen. Diese Abnahme wird jedoch später durch den Einfluss der zweiten Wachstumsrate abgemildert.

$\mathrm{Zu}$ untersuchen ist, wie sich der entgegengesetzte Einfluss der beiden Konjunkturvariablen auf die Beschäftigungsstabilität von Männern erklären lässt. Zudem ist herauszufinden, warum bei Frauen ein solch entgegengesetzter Einfluss nicht zu erkennen ist. Makroökonomische Untersuchungen haben ergeben, dass sich die Entwicklung von arbeitgeberseitigen Entlassungen und arbeitnehmerseitigen Kündigungen im Konjunkturverlauf unterscheidet. Das Entlassungsverhalten ist demnach antizyklisch, so dass in einem Boom weniger Arbeitskräfte entlassen werden als in einer Rezession. Das Kündigungsverhalten ist hingegen als prozyklisch beschrieben worden. Kündigungen seitens der Arbeitnehmer werden vermehrt in einem wirtschaftlichen Aufschwung vorgenommen. Ein solch entgegengesetztes Entlassungs- und Kündigungsverhalten könnte Ursache für die hier ermittelten Ergebnisse sein, wenn Kündigungen bei Männern im Aufschwung verzögert einsetzen und sich Kündigungen und Entlassungen bei Frauen im Laufe einer Aufschwungsphase ausgleichen. Um dies zu untersuchen, soll im Folgenden zwischen Beschäftigungsverhältnissen, die durch eine Entlassung enden, und Beschäftigungsverhältnissen, die vom Arbeitnehmer selbst durch eine Kündigung beendet werden, unterschieden werden.

Wenn ausschließlich Kündigungen oder Entlassungen betrachtet werden, ist zunächst zu fragen, ob weiterhin von einer langfristig fallenden Hazardrate ausgegangen werden kann. Dies ist zu bezweifeln, da bei einer solchen Eingrenzung des Datensatzes lang andauernde, stabile Beschäftigungsverhältnisse aus der Untersuchung ausscheiden. Es verbleiben lediglich Beschäftigungsverhältnisse, von denen bekannt ist, dass sie zumeist nur kurz- oder 
mittelfristig bestehen. Da sowohl Arbeitgeber als auch Arbeitnehmer sich bei ihren Entscheidungen über Entlassungen beziehungsweise Kündigungen von mehreren, üblicherweise nicht beobachtbaren Einflüssen leiten lassen, die sich im Zeitablauf als Erfahrungswert kumulieren, muss von einer im Zeitverlauf konstanten oder gar steigenden Hazardrate ausgegangen werden. Da das log-logistische AFT-Modell diesen Verlauf des Risikos einer Beschäftigungsbeendigung nicht abbilden kann, soll an dieser Stelle das Weibull-AFTModell herangezogen werden. In Tabelle 4 sind die Schätzergebnisse für die konjunkturellen Einflüsse zusammengefasst. Bei diesen Ergebnissen wird nicht für den Einfluss unbeobachteter Heterogenität kontrolliert, da sich die Varianz $\psi$ - vgl. Gleichung (4) - in keinem der Modelle als signifikant von Null verschieden erwies.

Für den Einfluss der Konjunkturvariablen lässt sich folgendes feststellen: In den Entlassungsmodellen ergeben sich bis auf eine Ausnahme sowohl für Männer als auch für Frauen positive Koeffizienten der beiden Wachstumsraten. Dies deutet im Gegensatz zu Garloff (2004) auf ein antizyklisches Entlassungsverhalten der Arbeitgeber hin, so dass bei hohen Wachstumsraten das Risiko einer Beschäftigungsbeendigung aufgrund einer Entlassung vergleichsweise gering ist. Allerdings sind nur die Koeffizienten der zweiten Wachstumsrate für beide Geschlechter leicht positiv signifikant. In den Kündigungsmodellen werden unabhängig vom Geschlecht für beide Konjunkturvariablen negative Koeffizienten geschätzt. Dies impliziert ein prozyklisches Kündigungsverhalten. Hier besteht Übereinstimmung mit den Ergebnissen aus aggregierten Daten des IAB-Betriebspanels für Baden-Württemberg bei Garloff (2004). Statistisch signifikant ist jedoch nur der Einfluss der ersten Wachstumsrate im Modell der Männer.

Die Schätzungen bestätigen also tendenziell die Ergebnisse makroökonomischer Untersuchungen hinsichtlich eines antizyklischen Entlassungsverhaltens und eines prozyklischen Kündigungsverhaltens. Sie liefern allerdings keinen Erklärungsbeitrag für die in Tabelle 3 dargestellten Ergebnisse, da nicht zu erkennen ist, dass Kündigungen bei Männern im Aufschwung verzögert einsetzen und sich Kündigungen und Entlassungen bei Frauen im Laufe einer Aufschwungsphase ausgleichen.

\section{$5 \quad$ Fazit}

Die Beschäftigungsstabilität im Konjunkturzyklus für Westdeutschland im Zeitraum von 1984 bis 2005 war Gegenstand dieses Beitrags. Dabei ist zunächst festzustellen, dass sich aus der mikroökonomischen Theorie kaum Erwartungen über den Zusammenhang zwischen der konjunkturellen Lage und der Dauer beziehungsweise der Stabilität von Beschäftigungsverhältnissen ableiten lassen. Makroökonomische Untersuchungen zeigen 
zwar, dass Mobilitätsprozesse auf dem Arbeitsmarkt zyklischen Schwankungen unterliegen, allerdings beruhen diese Ergebnisse auf aggregierten Daten, die keine differenzierte Analyse der Bestimmungsgründe der Beschäftigungsstabilität erlauben. Im empirischen Teil dieses Beitrags wurde daher ein auf Basis des Sozio-ökonomischen Panels erzeugter mikroökonomischer Datensatz eingesetzt, erweitert um zwei makroökonomische Variablen zur Erfassung der konjunkturellen Entwicklung. Dieser Datensatz wurde mit parametrischen Verfahren der Ereignisanalyse ausgewertet. Auf Basis des log-logistischen AFT-Modells lässt sich sowohl für Frauen als auch für Männer ein Einfluss der konjunkturellen Entwicklung auf die Beschäftigungsstabilität erkennen. Die Beschäftigungsstabilität nimmt für beide Geschlechter zu Beginn eines wirtschaftlichen Aufschwungs zu. Mit zunehmender Dauer des Aufschwungs setzt allerdings für Männer ein entgegengesetzter Einfluss ein und das Risiko einer Beschäftigungsbeendigung steigt aufgrund der hohen Wachstumsraten der Vorperioden.

In Zukunft muss verstärkt unter Verwendung größerer Datensätze untersucht werden, inwieweit sich die hier ermittelten Ergebnisse mit anderen Modellspezifikationen und für spezielle Untergruppen bestätigen lassen. 


\section{Literaturverzeichnis}

Akerlof, G.A., Rose, A.K., Yellen, J.L., Ball, L. und R.E. Hall (1988): "Job Switching and Job Satisfaction in the U.S. Labor Market", Brookings Papers on Economic Activity 2, S. 495-594.

Allison, P. (1982): "Discrete Time Methods for the Analysis of Event Histories", in: Leinhardt, S. (Hrsg.), Sociological Methodology, San Francisco, S. 61-98.

Arbeitskreis "Volkswirtschaftliche Gesamtrechnung der Länder" (2006): "Ergebnisse und Rückrechnungsergebnisse vor der Revision 2005: Bruttoinlandsprodukt in Preisen von 1995, 1970 bis 2004“, Download von http://www.vgrdl.de am 05.09.2006.

Barlevy, G. (2002): "The Sullying Effect of Recessions", Review of Economic Studies 69, S. 65-96.

Barrett, N.S. und B. Södersten (1975): "Unemployment Flows, Welfare and Labor Market Efficiency in Sweden and the United States", Swedish Journal of Economics, 77, S. 289 302.

Becker, G. (1962): "Investment in Human Capital: A Theoretical Analysis", Journal of Political Economy, 70, S. S9-S49.

Bergemann, A. (2004): "Labor Market Dynamics and Policy Evaluation: Empirical Evidence from Micro Data“, Dissertation an der Universität Mannheim, Download von http://bibserv7.bib.u mannheim.de/madoc/volltexte/2005/863/ am 05.09.2006.

Bergemann, A. und A. Mertens (2001): "Job Stability Trends, Layoffs and Quits - An Empirical Analysis for West Germany", Humboldt-Universität zu Berlin, Sonderforschungsbereich 373, Discussion Paper 102/2001.

Bergemann, A. und H. Schneider (1998): "Ist der deutsche Arbeitsmarkt beweglicher geworden? - Eine Analyse anhand der Unternehmenszugehörigkeitsdauer in Westdeutschland“, Wirtschaft im Wandel 11/1998, Institut für Wirtschaftsforschung Halle.

Blanchard, O. und P. Diamond (1992): "The Flow Approach to Labor Markets", American Economic Review, 82, S. 354-359.

Blanchard, O., Diamond, P., Hall, R.E. und K. Murphy (1990): "The Cyclical Behavior of the Gross Flows of U.S. Workers", Brookings Papers on Economic Activity 2, S. 85-155.

Boisjoly, J., Duncan, G. und T. Smeeding (1998): "The Shifting Incidence of Involuntary Job Losses from 1968 to 1992“, Industrial Relations, 37, S. 207-231.

Boockmann, B. und S. Steffes (2005): "Individual and plant-level Determinants of Job Duration in Germany“, ZEW Discussion Paper No. 05-89.

Booth, A., Francesconi, M. und C. Garcia-Serrano (1999): "Job Tenure and Job Mobility in Britain“, Industrial and Labor Relations Review, 53, S. 43-70.

Burda, M. (1993): "Decentralized Trade as a Market Imperfection: The Case of the Labor 
Market", in: B. Gahlen, H. Hesse und H.J. Ramser (Hrsg.), Makroökonomik unvollkommener Märkte, Tübingen: Mohr Siebeck, S. 181-197.

Burda, M. und C. Wyplosz (1994): "Gross Worker and Job Flows in Europe," European Economic Review, 38, S. 1287-1315.

Burgess, S. und H. Rees (1996): "Job Tenure in Britain 1975-1992", The Economic Journal, 106, S. 334-344.

Burgess, S. und H. Rees (1998): "A Disaggregate Analysis of the Evolution of Job Tenure in Britain, 1975-1993“, British Journal of Industrial Relations, 36, S. 629-657.

Caballero, R.J. und M.L. Hammour (1996): "On the Timing and Efficieny of Creative Destruction“, Quarterly Journal of Economics 111, 805-852.

Cameron, A. und P. Trivedi (2005): "Microeconometrics. Methods and Applications", Cambridge: Cambridge University Press.

Cleves, M., Gould, W. und R. Gutierrez (2004): "An Introduction to Survival Analysis Using Stata", Revised Edition, College Station/Texas: Stata Press.

Diebold, F., Neumark, D. und D. Polsky (1997): "Job Stability in the United States", Journal of Labor Economics, 15, S. 206-233.

Erlinghagen, M. (2002): "Die Entwicklung von Arbeitsmarktmobilität und Beschäftigungsstabilität im Übergang von der Industrie- zur Dienstleistungsgesellschaft", Mitteilungen aus der Arbeitsmarkt- und Berufsforschung, 35, S. 74-89.

Erlinghagen, M. (2004): "Die Restrukturierung des Arbeitsmarktes: Arbeitsmarktmobilität und Beschäftigungsstabilität im Zeitverlauf, Wiesbaden: VS Verlag für Sozialwissenschaften.

Erlinghagen, M. und M. Knuth (2004): "Beschäftigungsstabilität in der Wissensgesellschaft", in: Struck, O. und C. Köhler (Hrsg.): Beschäftigungsstabilität im Wandel? Empirische Befunde und theoretische Erklärungen für West- und Ostdeutschland, München: Rainer Hampp Verlag, S. 23-38.

Farber, H. (1995): "Are Lifetime Jobs Disappearing? Job Duration in the United States: 1973-1993“, NBER Working Paper No. 5014.

Garloff, A. (2004): "Arbeitsmarktdynamik und ex-post Mismatch in Baden-Württemberg: Eine deskriptive Analyse der Arbeitsplatz- und Arbeitskräftefluktuation", in: C. Arndt und H. Strotmann, Paneluntersuchungen als Instrument zur Analyse der Bestimmungsfaktoren des Strukturwandels, IAW-Forschungsberichte Bd. 63, Tübingen, S. 47-74.

Gerlach, K. und G. Stephan (2008): "A Note on Individual Tenure and Collective Contracts“, Labour, 22, S.167-183.

Gottschalk, P. und R. Moffitt (1999): "Changes in Job Stability and Insecurity Using Monthly Survey Data“, Journal of Labor Economics, 17, S91-S126. 
Gregg, P. und J. Wadsworth (1995): "A Short History of Labour Turnover, Job Tenure and Job Security, 1975-1993“, Oxford Review of Economic Policy, 11, S. 73-90.

Gregg, P. und J. Wadsworth (2002): "Job Tenure in Britain, 1975-2000: Is a Job for Life or Just for Christmas?", Oxford Bulletin of Economics and Statistics, 64, S. 111-134.

Grotheer, M. und O. Struck (2003): "Beschäftigungsstabilität: Entwicklung und Arbeitszufriedenheit", Mitteilungen aus der Arbeitsmarkt- und Berufsforschung, 3/2003, S. 300-328.

Gutierrez, R. (2002): "Parametric frailty and shared frailty survival models", The Stata Journal, 2, S. 22-44.

Hübler, O. (1989): "Ökonometrie“, Stuttgart: Gustav Fischer Verlag.

Hübler, O. (1993): "Korreferat zum Referat M. Burda", in: B. Gahlen, H. Hesse und H.J. Ramser (Hrsg.), Makroökonomik unvollkommener Märkte, Tübingen: Mohr Siebeck, S. 199-205.

Hujer, R., Mauer, K.O. und M. Wellner (1999): "Analyzing the Effects of On-the-job vs. Off-the-job Training on Unemployment Duration in West Germany", in: L. Bellmann und V. Steiner (Hrsg.), Panelanalysen zu Lohnstruktur, Qualifikation und Beschäftigungsdynamik, BeitAB 229, S. 203-237.

Jaeger, D. und A. Stevens (1999): "Is Job Stability in the United States Falling? Reconciling Trends in the Current Population Survey and Panel Study of Income Dynamics", Journal of Labor Economics, 17, S. S1-S28.

Jovanovic, B. (1979): "Job Matching and the Theory of Turnover", Journal of Political Economy, 87, S. 972-990.

Jürges, H. (2005): "Retrospective Error in the SOEP calendar Data: The Case of Unemployment", Schmollers Jahrbuch, 125, S. 157-165.

Krause, M.U. und T.A. Lubik (2006): "The Cyclical Upgrading of Labor and On-the-job Search“, Labour Economics, 13, 459-477.

Lee, E. und J. Wang (2003): "Statistical Methods for Survival Data Analysis", 3. Auflage, Hoboken/New Jersey: John Wiley \& Sons.

Mertens, A. (1999): "Job Stability Trends and Labor Market (Re-)Entry in West Germany 1984-1997“, Humboldt-Universität zu Berlin, Sonderforschungsbereich 373, Discussion Paper 60/1999.

Mincer, J. (1958): "Investment in Human Capital and Personal Income Distribution", Journal of Political Economy, 66, S. 281-302.

Mortensen, D.T. (1986): "Job Search and the Labor Market", in: O. Ashenfelter und R. Layard (eds.), Handbook of Labor Economics, Vol. II, Amsterdam: North-Holland, S. 849-919.

Mortensen, D.T. und C.A. Pissarides (1994): "Job Creation and Job Destruction in the 
Theory of Unemployment", Review of Economic Studies, 61, S. 397-415 .

Mortensen, D.T. und C.A. Pissarides (1999): "New Developments in Models of Search in the Labor Market", in: O. Ashenfelter und D. Card (eds.), Handbook of Labor Economics, Vol. 3B, Amsterdam: Elsevier, S. 2567-2627.

Moulton, B. (1986): "Random Group Effects and the Precision of Regression Estimates", Journal of Econometrics, 32, S. 385-397.

Moulton, B. (1990): "An Illustration of a Pitfall in Estimating the Effects of Aggregate Variables on Micro Units", The Review of Economics and Statistics, 72, S. 334-338.

Neumark, D., Polsky, D. und D. Hansen (1999): "Has Job Stability Declined Yet? New Evidence for the 1990s“, Journal of Labor Economics, 17, S. S29-S64.

Nierhaus, W. (1999): "Aus dem Instrumentenkasten der Konjunkturanalyse: Veränderungsraten im Vergleich“, Ifo-Schnelldienst 27/99, S. 11-19.

Pepper, J.V. (2002): "Robust Inferences from Random Clustered Samples: An Application Using Data from the Panel Study of Income Dynamics“, Economics Letters 75, S. 341-345.

Rose, S. (1995): "Declining Job Security and the Professionalization of Opportunity", Research Report No. 95-04, Washington: National Commission for Employment Policy.

Schasse, U. (1991): "Betriebszugehörigkeitsdauer und Mobilität. Eine empirische Untersuchung zur Stabilität von Beschäftigungsverhältnissen“, Frankfurt am Main: Campus Verlag.

Schettkat, R. (1996): "Flows in Labor Markets. Concepts and International Comparative Results" in: Schettkat, R. (Hrsg.): The Flow Analysis of Labour Markets, London: Routledge, S. 14-36.

Shimer, (2005):"The Cyclical Behavior of Equilibrium Unemployment and Vacancies", American Economic Review, 95, S. 25-49.

Winkelmann, R. und K. Zimmermann (1998): "Is job stability declining in Germany? Evidence from count data models", Applied Economics, 30, S. 1413-1420.

Winker, P. (2007): "Empirische Wirtschaftsforschung und Ökonometrie“, Berlin: Springer. 
Tabelle 1a Deskriptive Statistiken für alle Beschäftigungsverhältnisse (BV) und solche mit einer Dauer von mehr als sechs Monaten

\begin{tabular}{|c|c|c|c|c|}
\hline \multirow[t]{2}{*}{ Variable } & \multicolumn{2}{|c|}{ Männer } & \multicolumn{2}{|r|}{ Frauen } \\
\hline & alle BV & $\mathrm{BV}>6$ Monate & alle BV & $\mathrm{BV}>6$ Monate \\
\hline \multicolumn{5}{|l|}{ Alter } \\
\hline 16 - 25 Jahre & $\begin{array}{c}0,2546 \\
(0,4357)\end{array}$ & $\begin{array}{c}0,2344 \\
(0,4237)\end{array}$ & $\begin{array}{c}0,2069 \\
(0,4051)\end{array}$ & $\begin{array}{c}0,2013 \\
(0,4010)\end{array}$ \\
\hline 26 - 35 Jahre & $\begin{array}{c}0,3767 \\
(0,4846)\end{array}$ & $\begin{array}{c}0,3910 \\
(0,4880)\end{array}$ & $\begin{array}{c}0,3236 \\
(0,4679)\end{array}$ & $\begin{array}{c}0,3209 \\
(0,4669)\end{array}$ \\
\hline 36 - 45 Jahre & $\begin{array}{c}0,2159 \\
(0,4115)\end{array}$ & $\begin{array}{c}0,2262 \\
(0,4184)\end{array}$ & $\begin{array}{c}0,2481 \\
(0,4319)\end{array}$ & $\begin{array}{c}0,2514 \\
(0,4339)\end{array}$ \\
\hline 46 - 55 Jahre & $\begin{array}{c}0,0984 \\
(0,2978)\end{array}$ & $\begin{array}{c}0,0943 \\
(0,2923)\end{array}$ & $\begin{array}{c}0,1522 \\
(0,3592)\end{array}$ & $\begin{array}{c}0,1551 \\
(0,3621)\end{array}$ \\
\hline 56 - 65 Jahre & $\begin{array}{c}0,0544 \\
(0,2269)\end{array}$ & $\begin{array}{c}0,0541 \\
(0,2262)\end{array}$ & $\begin{array}{c}0,0692 \\
(0,2538)\end{array}$ & $\begin{array}{c}0,0712 \\
(0,2572)\end{array}$ \\
\hline \multicolumn{5}{|l|}{ Schulbildung } \\
\hline Hauptschule & $\begin{array}{c}0,5190 \\
(0,4997)\end{array}$ & $\begin{array}{c}0,5184 \\
(0,4997)\end{array}$ & $\begin{array}{c}0,4075 \\
(0,4914)\end{array}$ & $\begin{array}{c}0,4001 \\
(0,4900)\end{array}$ \\
\hline Realschule & $\begin{array}{c}0,1705 \\
(0,3761)\end{array}$ & $\begin{array}{c}0,1668 \\
(0,3728)\end{array}$ & $\begin{array}{c}0,3281 \\
(0,4696)\end{array}$ & $\begin{array}{c}0,3378 \\
(0,4730)\end{array}$ \\
\hline Fachhochschulreife & $\begin{array}{c}0,0681 \\
(0,2520)\end{array}$ & $\begin{array}{c}0,0702 \\
(0,2555)\end{array}$ & $\begin{array}{c}0,0470 \\
(0,2117)\end{array}$ & $\begin{array}{c}0,0474 \\
(0,2125)\end{array}$ \\
\hline Abitur & $\begin{array}{c}0,1692 \\
(0,3750)\end{array}$ & $\begin{array}{c}0,1750 \\
(0,3800)\end{array}$ & $\begin{array}{c}0,1486 \\
(0,3557)\end{array}$ & $\begin{array}{c}0,1464 \\
(0,3536)\end{array}$ \\
\hline Kein Abschluss & $\begin{array}{c}0,0731 \\
(0,2604)\end{array}$ & $\begin{array}{c}0,0696 \\
(0,2546)\end{array}$ & $\begin{array}{c}0,0687 \\
(0,2531)\end{array}$ & $\begin{array}{c}0,0683 \\
(0,2522)\end{array}$ \\
\hline \multicolumn{5}{|l|}{ Stellung im Beruf } \\
\hline Ungelernte(r) Arbeiter(in) & $\begin{array}{c}0,0597 \\
(0,2369)\end{array}$ & $\begin{array}{c}0,0541 \\
(0,2262)\end{array}$ & $\begin{array}{c}0,0886 \\
(0,2843)\end{array}$ & $\begin{array}{c}0,0849 \\
(0,2787)\end{array}$ \\
\hline Angelernte(r) Arbeiter(in) & $\begin{array}{c}0,1882 \\
(0,3909)\end{array}$ & $\begin{array}{c}0,1840 \\
(0,3876)\end{array}$ & $\begin{array}{c}0,1452 \\
(0,3523)\end{array}$ & $\begin{array}{c}0,1415 \\
(0,3486)\end{array}$ \\
\hline Gelernte(r) Facharbeiter(in) & $\begin{array}{c}0,2986 \\
(0,4577)\end{array}$ & $\begin{array}{c}0,2959 \\
(0,4565)\end{array}$ & $\begin{array}{c}0,0465 \\
(0,2108)\end{array}$ & $\begin{array}{c}0,0472 \\
(0,2120)\end{array}$ \\
\hline Vorarbeiter(in)/Meister(in) & $\begin{array}{c}0,0637 \\
(0,2442)\end{array}$ & $\begin{array}{c}0,0629 \\
(0,2427)\end{array}$ & $\begin{array}{c}0,0047 \\
(0,0688)\end{array}$ & $\begin{array}{c}0,0050 \\
(0,0703)\end{array}$ \\
\hline $\begin{array}{l}\text { Angestellte(r) mit einfacher } \\
\text { Tätigkeit }\end{array}$ & $\begin{array}{c}0,0769 \\
(0,2664)\end{array}$ & $\begin{array}{c}0,0776 \\
(0,2675)\end{array}$ & $\begin{array}{c}0,2662 \\
(0,4420)\end{array}$ & $\begin{array}{c}0,2611 \\
(0,4393)\end{array}$ \\
\hline
\end{tabular}


Fortsetzung von Tabelle 1a:

\begin{tabular}{|c|c|c|c|c|}
\hline \multirow[t]{2}{*}{ Variable } & \multicolumn{2}{|c|}{ Männer } & \multicolumn{2}{|r|}{ Frauen } \\
\hline & alle BV & $\mathrm{BV}>6$ Monate & alle BV & $\mathrm{BV}>6$ Monate \\
\hline Angestellte(r) mit qualifizierter & 0,1500 & 0,1523 & 0,3611 & 0,3733 \\
\hline Tätigkeit & $(0,3571)$ & $(0,3594)$ & $(0,4804)$ & $(0,4837)$ \\
\hline Angestellte(r) mit hochquali- & 0,1630 & 0,1733 & 0,0875 & 0,0871 \\
\hline fizierter Tätigkeit & $(0,3694)$ & $(0,3785)$ & $(0,2826)$ & $(0,2820)$ \\
\hline Teilzeit & 0,0637 & 0,0563 & 0,4247 & 0,4247 \\
\hline & $(0,2442)$ & $(0,2306)$ & $(0,4944)$ & $(0,4944)$ \\
\hline Öffentlicher Dienst & 0,1048 & 0,1065 & 0,2143 & 0,2182 \\
\hline & $(0,3064)$ & $(0,3085)$ & $(0,4104)$ & $(0,4131)$ \\
\hline \multicolumn{5}{|l|}{ Firmengröße } \\
\hline \multirow[t]{2}{*}{1 - 19 Beschäftigte } & 0,0799 & 0,0801 & 0,1153 & 0,1149 \\
\hline & $(0,2711)$ & $(0,2715)$ & $(0,3195)$ & $(0,3190)$ \\
\hline \multirow[t]{2}{*}{20 - 199 Beschäftigte } & 0,3632 & 0,3664 & 0,3946 & 0,3924 \\
\hline & $(0,4810)$ & $(0,4819)$ & $(0,4888)$ & $(0,4883)$ \\
\hline \multirow[t]{2}{*}{200 - 1999 Beschäftigte } & 0,0444 & 0,0484 & 0,0498 & 0,0509 \\
\hline & $(0,2061)$ & $(0,2147)$ & $(0,2175)$ & $(0,2198)$ \\
\hline \multirow[t]{2}{*}{2000 und mehr Beschäftigte } & 0,2516 & 0,2480 & 0,2221 & 0,2194 \\
\hline & $(0,4340)$ & $(0,4319)$ & $(0,4157)$ & $(0,4139)$ \\
\hline \multicolumn{5}{|l|}{ Wirtschaftssektor } \\
\hline \multirow[t]{2}{*}{ Produzierendes Gewerbe } & 0,3590 & 0,3582 & 0,1931 & 0,1914 \\
\hline & $(0,4798)$ & $(0,4795)$ & $(0,3948)$ & $(0,3934)$ \\
\hline \multirow[t]{2}{*}{ Baugewerbe } & 0,1975 & 0,1866 & 0,0224 & 0,0223 \\
\hline & $(0,3981)$ & $(0,3896)$ & $(0,1480)$ & $(0,1478)$ \\
\hline \multirow[t]{2}{*}{ Handel, Gastgewerbe, Verkehr } & 0,2174 & 0,2228 & 0,2806 & 0,2745 \\
\hline & $(0,4125)$ & $(0,4162)$ & $(0,4494)$ & $(0,4463)$ \\
\hline \multirow[t]{2}{*}{ Finanzierung und Vermietung } & 0,0260 & 0,0266 & 0,0479 & 0,0486 \\
\hline & $(0,1590)$ & $(0,1610)$ & $(0,2137)$ & $(0,2152)$ \\
\hline \multirow[t]{2}{*}{ Dienstleistungen } & 0,2002 & 0,2058 & 0,4559 & 0,4631 \\
\hline & $(0,4002)$ & $(0,4044)$ & $(0,4981)$ & $(0,4987)$ \\
\hline \multirow{2}{*}{$\begin{array}{l}\text { Dauer der Beschäftigungsver- } \\
\text { hältnisse }\end{array}$} & 37,2312 & 41,4513 & 33,5552 & 36,18218 \\
\hline & $(45,1611)$ & $(46,2940)$ & $(39,7240)$ & $(40,3650)$ \\
\hline \multirow{2}{*}{$\begin{array}{l}\text { Anzahl Beschäftigungsverhält- } \\
\text { nisse } \\
\text { Anzahl Personen }\end{array}$} & 4006 & 3532 & 4422 & 4029 \\
\hline & 2116 & 2002 & 2397 & 2320 \\
\hline \multicolumn{5}{|c|}{$\begin{array}{c}\text { Dargestellt sind Mittelwerte und Standardabweichungen darunter in Klammern. } \\
\text { Die Angaben beziehen sich auf den Endzeitpunkt der jeweils } \\
\text { zugrundeliegenden Beschäftigungsverhältnisse. }\end{array}$} \\
\hline
\end{tabular}


Tabelle 1b Deskriptive Statistiken für Kündigungen (K) und Entlassungen (E)

\begin{tabular}{|c|c|c|c|c|}
\hline \multirow[t]{2}{*}{ Variable } & \multicolumn{2}{|c|}{ Männer } & \multicolumn{2}{|c|}{ Frauen } \\
\hline & $\mathrm{K}$ & $\mathrm{E}$ & $\mathrm{K}$ & $\mathrm{E}$ \\
\hline \multicolumn{5}{|l|}{ Alter } \\
\hline 16 - 25 Jahre & $\begin{array}{c}0,2851 \\
(0,4518)\end{array}$ & $\begin{array}{c}0,3463 \\
(0,4770)\end{array}$ & $\begin{array}{c}0,3599 \\
(0,4804)\end{array}$ & $\begin{array}{c}0,3289 \\
(0,4714)\end{array}$ \\
\hline 26 - 35 Jahre & $\begin{array}{c}0,4971 \\
(0,5004)\end{array}$ & $\begin{array}{c}0,3659 \\
(0,4828)\end{array}$ & $\begin{array}{c}0,3298 \\
(0,4706)\end{array}$ & $\begin{array}{c}0,3355 \\
(0,4737)\end{array}$ \\
\hline 36 - 45 Jahre & $\begin{array}{c}0,1754 \\
(0,3806)\end{array}$ & $\begin{array}{c}0,1463 \\
(0,3543)\end{array}$ & $\begin{array}{c}0,2287 \\
(0,4204)\end{array}$ & $\begin{array}{c}0,1447 \\
(0,3530)\end{array}$ \\
\hline 46 - 55 Jahre & $\begin{array}{c}0,0322 \\
(0,1766)\end{array}$ & $\begin{array}{c}0,1024 \\
(0,3040)\end{array}$ & $\begin{array}{c}0,0603 \\
(0,2382)\end{array}$ & $\begin{array}{c}0,1250 \\
(0,3318)\end{array}$ \\
\hline 56 - 65 Jahre & $\begin{array}{c}0,0102 \\
(0,1007)\end{array}$ & $\begin{array}{c}0,0390 \\
(0,1941)\end{array}$ & $\begin{array}{c}0,0213 \\
(0,1444)\end{array}$ & $\begin{array}{c}0,0658 \\
(0,2487)\end{array}$ \\
\hline \multicolumn{5}{|l|}{ Schulbildung } \\
\hline Hauptschule & $\begin{array}{c}0,4781 \\
(0,4999)\end{array}$ & $\begin{array}{c}0,5610 \\
(0,4975)\end{array}$ & $\begin{array}{c}0,3741 \\
(0,4843)\end{array}$ & $\begin{array}{c}0,4276 \\
(0,4964)\end{array}$ \\
\hline Realschule & $\begin{array}{c}0,1857 \\
(0,3891)\end{array}$ & $\begin{array}{c}0,1854 \\
(0,3895)\end{array}$ & $\begin{array}{c}0,3582 \\
(0,4799)\end{array}$ & $\begin{array}{c}0,3421 \\
(0,4760)\end{array}$ \\
\hline Fachhochschulreife & $\begin{array}{c}0,0731 \\
(0,2605)\end{array}$ & $\begin{array}{c}0,0390 \\
(0,1941)\end{array}$ & $\begin{array}{c}0,0514 \\
(0,2210)\end{array}$ & $\begin{array}{c}0,0526 \\
(0,2240)\end{array}$ \\
\hline Abitur & $\begin{array}{c}0,2047 \\
(0,4038)\end{array}$ & $\begin{array}{c}0,0878 \\
(0,2837)\end{array}$ & $\begin{array}{c}0,1578 \\
(0,3649)\end{array}$ & $\begin{array}{c}0,1250 \\
(0,3318)\end{array}$ \\
\hline Kein Abschluss & $\begin{array}{c}0,0585 \\
(0,2348)\end{array}$ & $\begin{array}{c}0,1268 \\
(0,3336)\end{array}$ & $\begin{array}{c}0,0585 \\
(0,2349)\end{array}$ & $\begin{array}{c}0,0526 \\
(0,2240)\end{array}$ \\
\hline \multicolumn{5}{|l|}{ Stellung im Beruf } \\
\hline Ungelernte(r) Arbeiter(in) & $\begin{array}{c}0,0395 \\
(0,1949)\end{array}$ & $\begin{array}{c}0,1024 \\
(0,3040)\end{array}$ & $\begin{array}{c}0,0532 \\
(0,2246)\end{array}$ & $\begin{array}{c}0,0987 \\
(0,2992)\end{array}$ \\
\hline Angelernte(r) Arbeiter(in) & $\begin{array}{c}0,1535 \\
(0,3607)\end{array}$ & $\begin{array}{c}0,2683 \\
(0,4442)\end{array}$ & $\begin{array}{c}0,1099 \\
(0,3131)\end{array}$ & $\begin{array}{c}0,1447 \\
(0,3530)\end{array}$ \\
\hline Gelernte(r) Facharbeiter(in) & $\begin{array}{c}0,2909 \\
(0,4545)\end{array}$ & $\begin{array}{c}0,3024 \\
(0,4604)\end{array}$ & $\begin{array}{c}0,0709 \\
(0,2569)\end{array}$ & $\begin{array}{c}0,0461 \\
(0,2103)\end{array}$ \\
\hline Vorarbeiter(in)/Meister(in) & $\begin{array}{c}0,0541 \\
(0,2264)\end{array}$ & $\begin{array}{c}0,0341 \\
(0,1820)\end{array}$ & $\begin{array}{c}0,0070 \\
(0,0840)\end{array}$ & $\begin{array}{c}0,0000 \\
(0,0000)\end{array}$ \\
\hline $\begin{array}{l}\text { Angestellte(r) mit einfacher Tä- } \\
\text { tigkeit }\end{array}$ & $\begin{array}{c}0,0892 \\
(0,2852)\end{array}$ & $\begin{array}{c}0,0780 \\
(0,2689)\end{array}$ & $\begin{array}{c}0,2943 \\
(0,4561)\end{array}$ & $\begin{array}{c}0,1974 \\
(0,3993)\end{array}$ \\
\hline
\end{tabular}


Fortsetzung von Tabelle 1b:

\begin{tabular}{|c|c|c|c|c|}
\hline \multirow[t]{2}{*}{ Variable } & \multicolumn{2}{|c|}{ Männer } & \multicolumn{2}{|c|}{ Frauen } \\
\hline & K & $\mathrm{E}$ & $\mathrm{K}$ & $\mathrm{E}$ \\
\hline Angestellte(r) mit qual. Tätigkeit & $\begin{array}{c}0,1857 \\
(0,3891)\end{array}$ & $\begin{array}{c}0,1317 \\
(0,3390)\end{array}$ & $\begin{array}{c}0,3901 \\
(0,4882)\end{array}$ & $\begin{array}{c}0,4079 \\
(0,4931)\end{array}$ \\
\hline \multirow{2}{*}{$\begin{array}{l}\text { Angestellte(r) mit hochqual. Tä- } \\
\text { tigkeit }\end{array}$} & 0,1871 & 0,0829 & 0,0745 & 0,1053 \\
\hline & $(0,3903)$ & $(0,2764)$ & $(0,2628)$ & $(0,3079)$ \\
\hline Teilzeit & $\begin{array}{c}0,0322 \\
(0,1766)\end{array}$ & $\begin{array}{c}0,0146 \\
(0,1204)\end{array}$ & $\begin{array}{c}0,3156 \\
(0,4652)\end{array}$ & $\begin{array}{c}0,2632 \\
(0,4418)\end{array}$ \\
\hline Öffentlicher Dienst & $\begin{array}{c}0,0892 \\
(0,2852)\end{array}$ & $\begin{array}{c}0,0537 \\
(0,2259)\end{array}$ & $\begin{array}{c}0,1525 \\
(0,3598)\end{array}$ & $\begin{array}{c}0,1118 \\
(0,3162)\end{array}$ \\
\hline \multicolumn{5}{|l|}{ Firmengröße } \\
\hline 1 - 19 Beschäftigte & $\begin{array}{c}0,0673 \\
(0,2506)\end{array}$ & $\begin{array}{c}0,1024 \\
(0,3040)\end{array}$ & $\begin{array}{c}0,0993 \\
(0,2993)\end{array}$ & $\begin{array}{c}0,1711 \\
(0,3778)\end{array}$ \\
\hline 20 - 199 Beschäftigte & $\begin{array}{c}0,3918 \\
(0,4885)\end{array}$ & $\begin{array}{c}0,4293 \\
(0,4962)\end{array}$ & $\begin{array}{c}0,4202 \\
(0,4940)\end{array}$ & $\begin{array}{c}0,4474 \\
(0,4989)\end{array}$ \\
\hline 200 - 1999 Beschäftigte & $\begin{array}{c}0,0249 \\
(0,1558)\end{array}$ & $\begin{array}{c}0,0341 \\
(0,1820)\end{array}$ & $\begin{array}{c}0,0266 \\
(0,1610)\end{array}$ & $\begin{array}{c}0,0132 \\
(0,1143)\end{array}$ \\
\hline 2000 und mehr Beschäftigte & $\begin{array}{c}0,3348 \\
(0,4723)\end{array}$ & $\begin{array}{c}0,3171 \\
(0,4665)\end{array}$ & $\begin{array}{c}0,2713 \\
(0,4450)\end{array}$ & $\begin{array}{c}0,2500 \\
(0,4344)\end{array}$ \\
\hline \multicolumn{5}{|l|}{ Wirtschaftssektor } \\
\hline Produzierendes Gewerbe & $\begin{array}{c}0,3173 \\
(0,4657)\end{array}$ & $\begin{array}{c}0,3415 \\
(0,4754)\end{array}$ & $\begin{array}{c}0,1684 \\
(0,3746)\end{array}$ & $\begin{array}{c}0,2039 \\
(0,4043)\end{array}$ \\
\hline Baugewerbe & $\begin{array}{c}0,1608 \\
(0,3676)\end{array}$ & $\begin{array}{c}0,2341 \\
(0,4245)\end{array}$ & $\begin{array}{c}0,0195 \\
(0,1384)\end{array}$ & $\begin{array}{c}0,0132 \\
(0,1143)\end{array}$ \\
\hline Handel, Gastgewerbe, Verkehr & $\begin{array}{c}0,2675 \\
(0,4430)\end{array}$ & $\begin{array}{c}0,2439 \\
(0,4305)\end{array}$ & $\begin{array}{c}0,3316 \\
(0,4712)\end{array}$ & $\begin{array}{c}0,3816 \\
(0,4874)\end{array}$ \\
\hline Finanzierung und Vermietung & $\begin{array}{c}0,0278 \\
(0,1645)\end{array}$ & $\begin{array}{c}0,0146 \\
(0,1204)\end{array}$ & $\begin{array}{c}0,0496 \\
(0,2174)\end{array}$ & $\begin{array}{c}0,0197 \\
(0,1396)\end{array}$ \\
\hline Dienstleistungen & $\begin{array}{c}0,2266 \\
(0,4189)\end{array}$ & $\begin{array}{c}0,1659 \\
(0,3729)\end{array}$ & $\begin{array}{c}0,4309 \\
(0,4956)\end{array}$ & $\begin{array}{c}0,3816 \\
(0,4874)\end{array}$ \\
\hline $\begin{array}{l}\text { Dauer der Beschäftigungsver- } \\
\text { hältnisse }\end{array}$ & $\begin{array}{l}31,4328 \\
(33,7832)\end{array}$ & $\begin{array}{c}25,4146 \\
(26,7332)\end{array}$ & $\begin{array}{c}25,2890 \\
(30,1856)\end{array}$ & $\begin{array}{r}29,3355 \\
(33,5326)\end{array}$ \\
\hline $\begin{array}{l}\text { Anzahl Beschäftigungsverhält- } \\
\text { nisse }\end{array}$ & 684 & 205 & 564 & 152 \\
\hline Anzahl Personen & 484 & 178 & 439 & 138 \\
\hline \multicolumn{5}{|c|}{$\begin{array}{c}\text { Dargestellt sind Mittelwerte und Standardabweichungen darunter in Klammern. } \\
\text { Die Angaben beziehen sich auf den Endzeitpunkt der jeweils } \\
\text { zugrundeliegenden Beschäftigungsverhältnisse. }\end{array}$} \\
\hline
\end{tabular}


Tabelle 2: Schätzungen für alle Beschäftigungsverhältnisse

\begin{tabular}{|c|c|c|c|c|}
\hline & \multicolumn{2}{|c|}{ Männer } & \multicolumn{2}{|c|}{ Frauen } \\
\hline & WB & LL & WB & LL \\
\hline $\begin{array}{l}\text { Durchschnitt der aktuellen und der } \\
\text { um } 1 \mathrm{Jahr} \text { verzögerten Wachstumsrate }\end{array}$ & $\begin{array}{c}0,0293 \\
(0,0369) \\
{[0,0316]}\end{array}$ & $\begin{array}{c}0,0730 \\
(0,0506) \\
{[0,0495]}\end{array}$ & $\begin{array}{c}0,0324 \\
(0,0319) \\
{[0,0409]}\end{array}$ & $\begin{array}{l}0,0755^{+} \\
(0,0416) \\
{[0,0436]}\end{array}$ \\
\hline $\begin{array}{l}\text { Durchschnitt der um } 2 \text { und } 3 \text { Jahre } \\
\text { verzögerten Wachstumsraten }\end{array}$ & $\begin{array}{c}-0,0875^{* *} \\
(0,0370) \\
{[0,0304]}\end{array}$ & $\begin{array}{l}-0,0548 \\
(0,0514) \\
{[0,0441]}\end{array}$ & $\begin{array}{c}0,0316 \\
(0,0314) \\
{[0,0262]}\end{array}$ & $\begin{array}{c}0,0329 \\
(0,0394) \\
{[0,0390]}\end{array}$ \\
\hline$\cdots$ & $\ldots$ & $\cdots$ & $\ldots$ & $\cdots$ \\
\hline Schätzungen für $\gamma$ & $\begin{array}{c}0,8059 \\
(0,0199) \\
{[0,0211]}\end{array}$ & & $\begin{array}{c}0,9878 \\
(0,0349) \\
{[0,0362]}\end{array}$ & \\
\hline Schätzungen für $\delta^{-1}$ & & $\begin{array}{c}0,6787 \\
(0,0133) \\
{[0,0197]}\end{array}$ & & $\begin{array}{c}0,5260 \\
(0,0093) \\
{[0,0153]}\end{array}$ \\
\hline Anzahl Beschäftigungsverhältnisse & 4006 & 4006 & 4422 & 4422 \\
\hline Anzahl Personen & 2116 & 2116 & 2397 & 2397 \\
\hline Log-Likelihood & $-2641,2743$ & $-2820,9108$ & $-2157,0774$ & $-2384,756$ \\
\hline $\begin{array}{r}\text { WB: Weibull AFT Mo } \\
\text { Koeffizienten-/Parameter } \\
\text { Heteroskedastierobuste } \\
\text { Heteroskedastie- und clusterr } \\
+^{+} ;{ }^{*} \text { bzw. }{ }^{* *} \text { zeigt Signifikanz der Koeff }\end{array}$ & $\begin{array}{l}\text { LL: log } \\
\text { chätzungen } \\
\text { Standardfeh } \\
\text { buste Stand } \\
\text { ienten auf e }\end{array}$ & $\begin{array}{l}\text { ogistisches } A \\
\text { asierend auf } \\
\text { rin runden } \\
\text { rdfehler in e } \\
\text { em Niveau }\end{array}$ & $\begin{array}{l}\text { FT Modell } \\
\text { Gleichung (1) } \\
\text { Klammern } \\
\text { kigen Klamm } \\
\text { ron } \alpha=0,1 ; 0\end{array}$ & $\begin{array}{l}\text { n } \\
5 \text { bzw. } 0,01\end{array}$ \\
\hline
\end{tabular}


Tabelle 3: Schätzungen für Beschäftigungsverhältnisse mit einer Dauer von mehr als sechs Monaten

\begin{tabular}{|c|c|c|c|c|}
\hline & \multicolumn{2}{|c|}{ Männer } & \multicolumn{2}{|c|}{ Frauen } \\
\hline & WB & LL & WB & LL \\
\hline $\begin{array}{l}\text { Durchschnitt der aktuellen und der } \\
\text { um } 1 \text { Jahr verzögerten Wachstumsrate }\end{array}$ & $\begin{array}{c}0,0332 \\
(0,0374) \\
{[0,0283]}\end{array}$ & $\begin{array}{c}0,0744^{+} \\
(0,0450) \\
{[0,0397]}\end{array}$ & $\begin{array}{c}0,0343 \\
(0,0321) \\
{[0,0400]}\end{array}$ & $\begin{array}{l}0,0640^{+} \\
(0,0394) \\
{[0,0366]}\end{array}$ \\
\hline $\begin{array}{l}\text { Durchschnitt der um } 2 \text { und } 3 \text { Jahre } \\
\text { verzögerten Wachstumsraten }\end{array}$ & $\begin{array}{c}-0,0983^{* *} \\
(0,0377) \\
{[0,0302]}\end{array}$ & $\begin{array}{c}-0,1063^{* *} \\
(0,0452) \\
{[0,0368]}\end{array}$ & $\begin{array}{c}0,0330 \\
(0,0317) \\
{[0,0260]}\end{array}$ & $\begin{array}{c}0,0305 \\
(0,0376) \\
{[0,0341]}\end{array}$ \\
\hline$\cdots$ & $\cdots$ & $\cdots$ & $\cdots$ & $\cdots$ \\
\hline Schätzungen für $\gamma$ & $\begin{array}{c}1,0204 \\
(0,0481) \\
{[0,0651]}\end{array}$ & & $\begin{array}{c}1,1507 \\
(0,0464) \\
{[0,0526]}\end{array}$ & \\
\hline Schätzungen für $\delta^{-1}$ & & $\begin{array}{c}0,5954 \\
(0,0129) \\
{[0,0154]}\end{array}$ & & $\begin{array}{c}0,4895 \\
(0,0092) \\
{[0,0130]}\end{array}$ \\
\hline Anzahl Beschäftigungsverhältnisse & 3532 & 3532 & 4029 & 4029 \\
\hline Anzahl Personen & 2002 & 2002 & 2320 & 2320 \\
\hline Log-Likelihood & $-2200,9159$ & $-2262,8612$ & $-1928,7817$ & $-2048,3887$ \\
\hline $\begin{array}{r}\text { WB: Weibull AFT Mo } \\
\text { Koeffizienten-/Parameter } \\
\text { Heteroskedastierobuste } \\
\text { Heteroskedastie- und clusterr } \\
{ }^{+} ;{ }^{*} \text { bzw. }{ }^{* *} \text { zeigt Signifikanz der Koeff }\end{array}$ & $\begin{array}{l}\text { LL: log } \\
\text { chätzungen } \\
\text { Standardfeh } \\
\text { buste Stand } \\
\text { ienten auf e }\end{array}$ & $\begin{array}{l}\text { ogistisches } A \\
\text { asierend auf } \\
\text { r in runden } \\
\text { rdfehler in e } \\
\text { em Niveau }\end{array}$ & $\begin{array}{l}\text { FT Modell } \\
\text { Gleichung }(1 \\
\text { Klammern } \\
\text { ckigen Klamr } \\
\text { ron } \alpha=0,1\end{array}$ & 05 bzw. 0,01 \\
\hline
\end{tabular}


Tabelle 4: Schätzungen für Kündigungen und Entlassungen

\begin{tabular}{|c|c|c|c|c|}
\hline & \multicolumn{2}{|c|}{ Männer } & \multicolumn{2}{|c|}{ Frauen } \\
\hline & K & E & K & $\mathrm{E}$ \\
\hline $\begin{array}{l}\text { Durchschnitt der aktuellen und der } \\
\text { um } 1 \text { Jahr verzögerten Wachstumsrate }\end{array}$ & $\begin{array}{c}-0,0591^{*} \\
(0,0268) \\
{[0,0247]}\end{array}$ & $\begin{array}{l}-0,0244 \\
(0,0399) \\
{[0,0337]}\end{array}$ & $\begin{array}{l}-0,0328 \\
(0,0289) \\
{[0,0230]}\end{array}$ & $\begin{array}{c}0,0051 \\
(0,0517) \\
{[0,0421]}\end{array}$ \\
\hline $\begin{array}{l}\text { Durchschnitt der um } 2 \text { und } 3 \text { Jahre } \\
\text { verzögerten Wachstumsraten }\end{array}$ & $\begin{array}{l}-0,0102 \\
(0,0266) \\
{[0,0263]}\end{array}$ & $\begin{array}{l}0,0594^{+} \\
(0,0358) \\
{[0,0307]}\end{array}$ & $\begin{array}{c}-0,0114 \\
(0,0295) \\
{[0,0254]}\end{array}$ & $\begin{array}{l}0,0930^{+} \\
(0,0485) \\
{[0,0495]}\end{array}$ \\
\hline$\ldots$ & $\ldots$ & $\ldots$ & $\ldots$ & $\ldots$ \\
\hline Schätzung für $\gamma$ & $\begin{array}{c}0,9353 \\
(0,0466) \\
{[0,0441]}\end{array}$ & $\begin{array}{c}1,2184 \\
(0,0943) \\
{[0,0850]}\end{array}$ & $\begin{array}{c}1,0510 \\
(0,0511) \\
{[0,0468]}\end{array}$ & $\begin{array}{c}1,0955 \\
(0,1067) \\
{[0,0872]}\end{array}$ \\
\hline Anzahl Beschäftigungsverhältnisse & 684 & 205 & 564 & 152 \\
\hline Anzahl Personen & 484 & 178 & 439 & 138 \\
\hline Log-Likelihood & $-199,4975$ & 20,2119 & $-61,8771$ & 1,4207 \\
\hline $\begin{array}{r}\text { K: Kündig } \\
\text { Koeffizienten-/Parameter } \\
\text { Heteroskedastierobuste } \\
{ }^{+} ;^{*} \text { bzw. }{ }^{* *} \text { zeigt Signifikanz der Koeff }\end{array}$ & $\begin{array}{l}\text { Ingen E: } \\
\text { chätzungen } \\
\text { Standardfe } \\
\text { buste Stan } \\
\text { ienten auf }\end{array}$ & $\begin{array}{l}\text { Dntlassung } \\
\text { oasierend } \\
\text { ler in run } \\
\text { ardfehler } \\
\text { inem Nive }\end{array}$ & $\begin{array}{l}\text { len Klamn } \\
\text { n eckigen } \\
\text { au von } \alpha\end{array}$ & $\begin{array}{l}\text { mern } \\
0,05 \mathrm{bzw}\end{array}$ \\
\hline
\end{tabular}


Anhang: Fortsetzung 1 von Tabelle 2:

\begin{tabular}{|c|c|c|c|c|}
\hline \multirow[t]{2}{*}{ Variable } & \multicolumn{2}{|c|}{ Männer } & \multicolumn{2}{|c|}{ Frauen } \\
\hline & WB & LL & WB & LL \\
\hline \multicolumn{5}{|l|}{ Alter (Basis $=26-35$ Jahre $)$} \\
\hline 16 - 25 Jahre & $\begin{array}{c}-0,3719^{* *} \\
(0,0662) \\
{[0,0722]}\end{array}$ & $\begin{array}{c}-0,3418^{* *} \\
(0,0862) \\
{[0,0988]}\end{array}$ & $\begin{array}{l}-0,0129 \\
(0,0593) \\
{[0,0600]}\end{array}$ & $\begin{array}{c}0,1057 \\
(0,0679) \\
{[0,0797]}\end{array}$ \\
\hline 36 - 45 Jahre & $\begin{array}{c}0,0193 \\
(0,0745) \\
{[0,0744]}\end{array}$ & $\begin{array}{l}-0,0065 \\
(0,1126) \\
{[0,1288]}\end{array}$ & $\begin{array}{c}0,4846^{* *} \\
(0,0583) \\
{[0,0613]}\end{array}$ & $\begin{array}{c}0,4746^{* *} \\
(0,0794) \\
{[0,0927]}\end{array}$ \\
\hline 46 - 55 Jahre & $\begin{array}{l}-0,1015 \\
(0,0921) \\
{[0,1163]}\end{array}$ & $\begin{array}{l}-0,0985 \\
(0,1310) \\
{[0,1833]}\end{array}$ & $\begin{array}{c}0,5820^{* *} \\
(0,0703) \\
{[0,0757]}\end{array}$ & $\begin{array}{c}0,7171^{\text {** }} \\
(0,0910) \\
{[0,0946]}\end{array}$ \\
\hline 56 - 65 Jahre & $\begin{array}{c}-0,4952^{* *} \\
(0,1148) \\
{[0,1120]}\end{array}$ & $\begin{array}{c}-0,7429^{* *} \\
(0,1854) \\
{[0,2172]}\end{array}$ & $\begin{array}{c}0,1114 \\
(0,0960) \\
{[0,0872]}\end{array}$ & $\begin{array}{c}-0,0252 \\
(0,1580) \\
{[0,1803]}\end{array}$ \\
\hline \multicolumn{5}{|c|}{ Schulbildung (Basis = Realschule) } \\
\hline Hauptschule & $\begin{array}{l}0,1226^{+} \\
(0,0740) \\
{[0,0645]}\end{array}$ & $\begin{array}{l}0,1929^{+} \\
(0,0980) \\
{[0,0995]}\end{array}$ & $\begin{array}{l}-0,0670 \\
(0,0554) \\
{[0,0589]}\end{array}$ & $\begin{array}{c}-0,1186 \\
(0,0682) \\
{[0,0745]}\end{array}$ \\
\hline Fachhochschulreife & $\begin{array}{c}0,3158^{*} \\
(0,1269) \\
{[0,1291]}\end{array}$ & $\begin{array}{c}0,4109^{*} \\
(0,1617) \\
{[0,2011]}\end{array}$ & $\begin{array}{l}-0,0083 \\
(0,1060) \\
{[0,1173]}\end{array}$ & $\begin{array}{c}0,0522 \\
(0,1342) \\
{[0,1274]}\end{array}$ \\
\hline Abitur & $\begin{array}{l}-0,0479 \\
(0,0989) \\
{[0,0956]}\end{array}$ & $\begin{array}{l}0,2599^{+} \\
(0,1380) \\
{[0,1387]}\end{array}$ & $\begin{array}{c}-0,2410^{* *} \\
(0,0699) \\
{[0,0768]}\end{array}$ & $\begin{array}{c}-0,2753^{* *} \\
(0,0919) \\
{[0,1044]}\end{array}$ \\
\hline Kein Abschluss & $\begin{array}{l}-0,1507 \\
(0,1127) \\
{[0,1155]}\end{array}$ & $\begin{array}{c}0,0594 \\
(0,1454) \\
{[0,1664]}\end{array}$ & $\begin{array}{l}-0,0560 \\
(0,0939) \\
{[0,0873]}\end{array}$ & $\begin{array}{c}-0,0601 \\
(0,1133) \\
{[0,1200]}\end{array}$ \\
\hline \multicolumn{5}{|c|}{$\begin{array}{l}\text { Stellung im Beruf (Basis = Ange- } \\
\text { stellte(r) mit qualifizierter Tätigkeit) }\end{array}$} \\
\hline Ungelernte(r) Arbeiter(in) & $\begin{array}{c}-0,7938^{* *} \\
(0,1277) \\
{[0,1410]}\end{array}$ & $\begin{array}{c}-0,8550^{* *} \\
(0,1713) \\
{[0,2041]}\end{array}$ & $\begin{array}{c}-0,4463^{* *} \\
(0,0873) \\
{[0,0969]}\end{array}$ & $\begin{array}{c}-0,4810^{* *} \\
(0,1043) \\
{[0,1238]}\end{array}$ \\
\hline Angelernte(r) Arbeiter(in) & $\begin{array}{c}-0,3849^{* *} \\
(0,0966) \\
{[0,0917]}\end{array}$ & $\begin{array}{c}-0,3443^{*} \\
(0,1297) \\
{[0,1441]}\end{array}$ & $\begin{array}{c}-0,3436^{* *} \\
(0,0755) \\
{[0,0788]}\end{array}$ & $\begin{array}{c}-0,3614^{* *} \\
(0,0929) \\
{[0,0981]}\end{array}$ \\
\hline Gelernte(r) Facharbeiter(in) & $\begin{array}{l}-0,1074 \\
(0,0917) \\
{[0,0901]}\end{array}$ & $\begin{array}{l}-0,0980 \\
(0,1202) \\
{[0,1417]}\end{array}$ & $\begin{array}{l}-0,1149 \\
(0,1039) \\
{[0,0961]}\end{array}$ & $\begin{array}{c}-0,1191 \\
(0,1174) \\
{[0,1117]}\end{array}$ \\
\hline
\end{tabular}


Fortsetzung 2 von Tabelle 2:

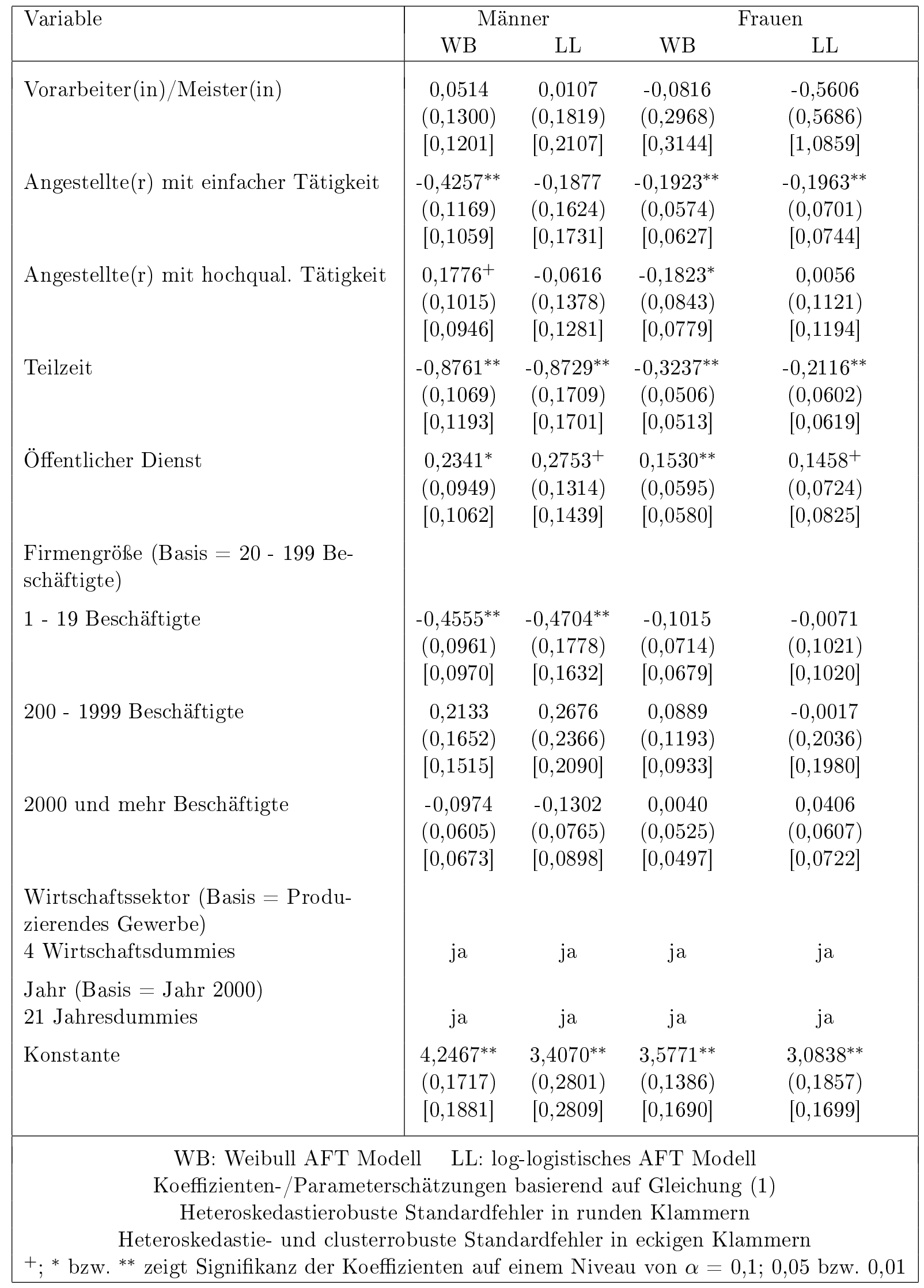


Anhang: Fortsetzung 1 von Tabelle 3:

\begin{tabular}{|c|c|c|c|c|}
\hline \multirow[t]{2}{*}{ Variable } & \multicolumn{2}{|c|}{ Männer } & \multicolumn{2}{|c|}{ Frauen } \\
\hline & WB & LL & WB & LL \\
\hline \multicolumn{5}{|l|}{ Alter (Basis $=26-35$ Jahre $)$} \\
\hline 16 - 25 Jahre & $\begin{array}{c}-0,2993^{* *} \\
(0,0662) \\
{[0,0700]}\end{array}$ & $\begin{array}{c}-0,2773^{* *} \\
(0,0755) \\
{[0,0872]}\end{array}$ & $\begin{array}{l}-0,0414 \\
(0,0587) \\
{[0,0601]}\end{array}$ & $\begin{array}{c}0,0313 \\
(0,0637) \\
{[0,0730]}\end{array}$ \\
\hline 36 - 45 Jahre & $\begin{array}{c}0,0404 \\
(0,0744) \\
{[0,0725]}\end{array}$ & $\begin{array}{c}0,0683 \\
(0,0975) \\
{[0,0974]}\end{array}$ & $\begin{array}{c}0,4566^{* *} \\
(0,0590) \\
{[0,0607]}\end{array}$ & $\begin{array}{c}0,4190^{* *} \\
(0,0737) \\
{[0,0838]}\end{array}$ \\
\hline 46 - 55 Jahre & $\begin{array}{c}0,0092 \\
(0,0947) \\
{[0,0934]}\end{array}$ & $\begin{array}{c}0,0422 \\
(0,1157) \\
{[0,1136]}\end{array}$ & $\begin{array}{c}0,5610^{* *} \\
(0,0709) \\
{[0,0720]}\end{array}$ & $\begin{array}{c}0,6704^{* *} \\
(0,0849) \\
{[0,0835]}\end{array}$ \\
\hline 56 - 65 Jahre & $\begin{array}{c}-0,5141^{* *} \\
(0,1252) \\
{[0,1159]}\end{array}$ & $\begin{array}{c}-0,4970^{* *} \\
(0,1579) \\
{[0,1453]}\end{array}$ & $\begin{array}{c}0,0768 \\
(0,1003) \\
{[0,0934]}\end{array}$ & $\begin{array}{c}0,0927 \\
(0,1448) \\
{[0,1516]}\end{array}$ \\
\hline \multicolumn{5}{|c|}{ Schulbildung (Basis = Realschule) } \\
\hline Hauptschule & $\begin{array}{c}0,0670 \\
(0,0741) \\
{[0,0682]}\end{array}$ & $\begin{array}{c}0,0674 \\
(0,0846) \\
{[0,0867]}\end{array}$ & $\begin{array}{l}-0,0314 \\
(0,0556) \\
{[0,0578]}\end{array}$ & $\begin{array}{c}-0,0723 \\
(0,0641) \\
{[0,0688]}\end{array}$ \\
\hline Fachhochschulreife & $\begin{array}{c}0,2552^{*} \\
(0,1241) \\
{[0,1246]}\end{array}$ & $\begin{array}{c}0,3114^{*} \\
(0,1397) \\
{[0,1471]}\end{array}$ & $\begin{array}{c}0,0256 \\
(0,1057) \\
{[0,1100]}\end{array}$ & $\begin{array}{c}0,0584 \\
(0,1256) \\
{[0,1215]}\end{array}$ \\
\hline Abitur & $\begin{array}{l}-0,0763 \\
(0,0994) \\
{[0,0882]}\end{array}$ & $\begin{array}{c}0,0933 \\
(0,1194) \\
{[0,0988]}\end{array}$ & $\begin{array}{c}-0,1745^{*} \\
(0,0702) \\
{[0,0739]}\end{array}$ & $\begin{array}{c}-0,0964 \\
(0,0850) \\
{[0,0949]}\end{array}$ \\
\hline Kein Abschluss & $\begin{array}{l}-0,1721 \\
(0,1152) \\
{[0,1161]}\end{array}$ & $\begin{array}{l}-0,0112 \\
(0,1263) \\
{[0,1334]}\end{array}$ & $\begin{array}{l}-0,0272 \\
(0,0945) \\
{[0,0863]}\end{array}$ & $\begin{array}{c}0,0084 \\
(0,1054) \\
{[0,1026]}\end{array}$ \\
\hline \multicolumn{5}{|c|}{$\begin{array}{l}\text { Stellung im Beruf (Basis = Ange- } \\
\text { stellte(r) mit qualifizierter Tätigkeit) }\end{array}$} \\
\hline Ungelernte(r) Arbeiter(in) & $\begin{array}{c}-0,7399^{* *} \\
(0,1363) \\
{[0,1375]}\end{array}$ & $\begin{array}{c}-0,8089^{* *} \\
(0,1567) \\
{[0,1549]}\end{array}$ & $\begin{array}{c}-0,3600^{* *} \\
(0,0890) \\
{[0,0926]}\end{array}$ & $\begin{array}{c}-0,3784^{* *} \\
(0,0992) \\
{[0,1102]}\end{array}$ \\
\hline Angelernte(r) Arbeiter(in) & $\begin{array}{c}-0,3616^{* *} \\
(0,0967) \\
{[0,0857]}\end{array}$ & $\begin{array}{c}-0,2799^{* *} \\
(0,1130) \\
{[0,1091]}\end{array}$ & $\begin{array}{c}-0,2599^{* *} \\
(0,0762) \\
{[0,0733]}\end{array}$ & $\begin{array}{c}-0,2349^{* *} \\
(0,0867) \\
{[0,0854]}\end{array}$ \\
\hline Gelernte(r) Facharbeiter(in) & $\begin{array}{c}-0,1408^{+} \\
(0,0906) \\
{[0,0787]}\end{array}$ & $\begin{array}{l}-0,1464 \\
(0,1059) \\
{[0,0977]}\end{array}$ & $\begin{array}{l}-0,1150 \\
(0,1022) \\
{[0,0898]}\end{array}$ & $\begin{array}{c}-0,1158 \\
(0,1126) \\
{[0,1077]}\end{array}$ \\
\hline
\end{tabular}


Fortsetzung 2 von Tabelle 3:

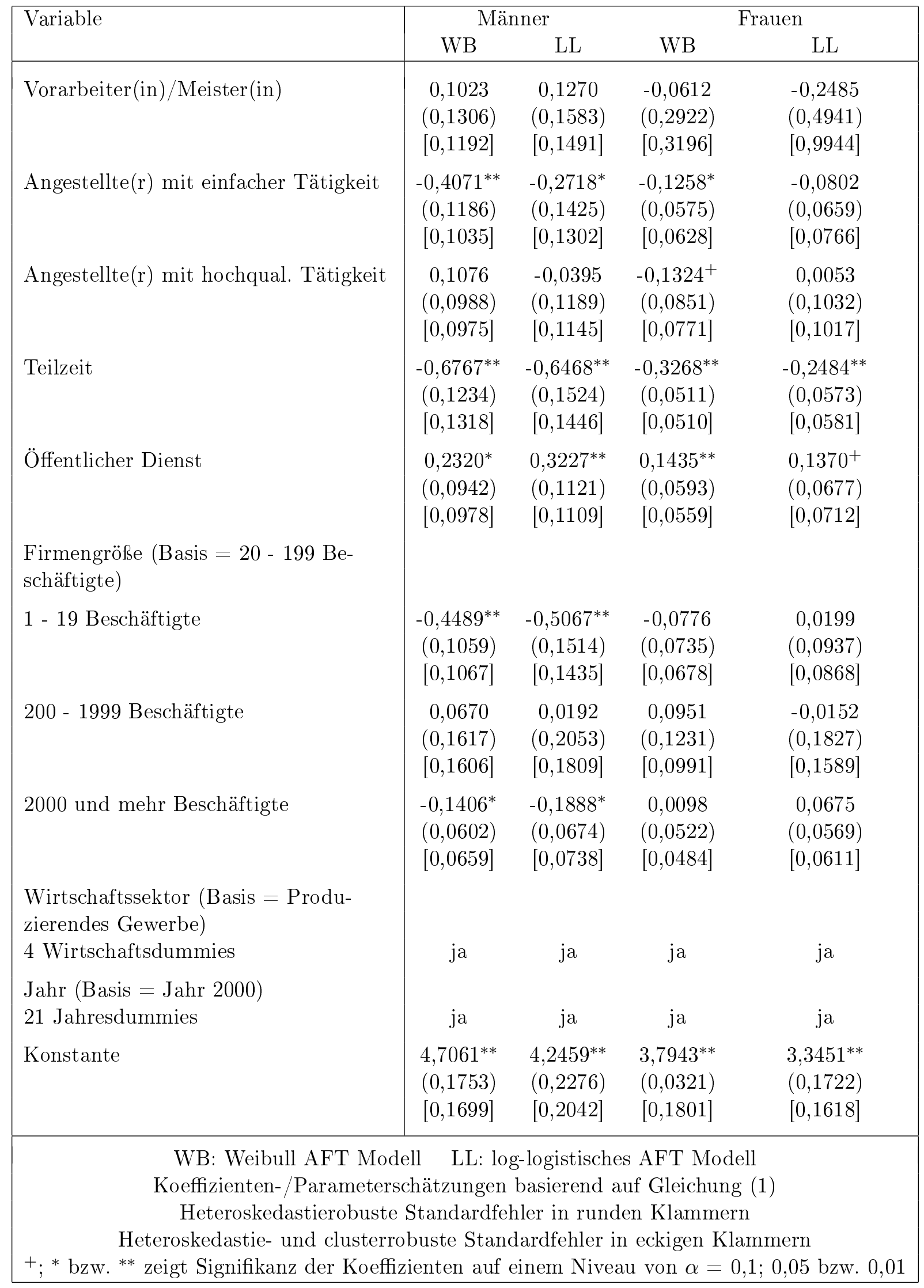


Anhang: Fortsetzung 1 von Tabelle 4:

\begin{tabular}{|c|c|c|c|c|}
\hline \multirow[t]{2}{*}{ Variable } & \multicolumn{2}{|c|}{ Männer } & \multicolumn{2}{|c|}{ Frauen } \\
\hline & K & E & K & $\mathrm{E}$ \\
\hline \multicolumn{5}{|l|}{ Alter (Basis $=26$ - 35 Jahre) } \\
\hline 16 - 25 Jahre & $\begin{array}{l}-0,0308 \\
(0,1097) \\
{[0,0945]}\end{array}$ & $\begin{array}{c}-0,4009^{* *} \\
(0,1549) \\
{[0,1517]}\end{array}$ & $\begin{array}{l}-0,1184 \\
(0,1070) \\
{[0,0926]}\end{array}$ & $\begin{array}{l}-0,2661 \\
{[0,2183]}\end{array}$ \\
\hline 36 - 45 Jahre & $\begin{array}{l}-0,0194 \\
(0,1186) \\
{[0,1091]}\end{array}$ & $\begin{array}{l}-0,0359 \\
(0,1943) \\
{[0,2042]}\end{array}$ & $\begin{array}{c}0,1063 \\
(0,1153) \\
{[0,1194]}\end{array}$ & $\begin{array}{r}0,4591^{+} \\
{[0,2459]}\end{array}$ \\
\hline 46 - 55 Jahre & $\begin{array}{c}-0,4756^{+} \\
(0,2427) \\
{[0,2470]}\end{array}$ & $\begin{array}{c}0,0595 \\
(0,2137) \\
{[0,1922]}\end{array}$ & $\begin{array}{c}0,4883^{* *} \\
(0,1881) \\
{[0,1790]}\end{array}$ & $\begin{array}{l}0,6937^{*} \\
{[0,2736]}\end{array}$ \\
\hline 56 - 65 Jahre & $\begin{array}{l}-0,1472 \\
(0,4271) \\
{[0,3858]}\end{array}$ & $\begin{array}{c}0,0227 \\
(0,3221) \\
{[0,3173]}\end{array}$ & $\begin{array}{c}0,1510 \\
(0,2947) \\
{[0,2421]}\end{array}$ & $\begin{array}{r}0,1175 \\
{[0,3161]}\end{array}$ \\
\hline \multicolumn{5}{|c|}{ Schulbildung (Basis $=$ Realschule) } \\
\hline Hauptschule & $\begin{array}{c}0,1233 \\
(0,1188) \\
{[0,1002]}\end{array}$ & $\begin{array}{l}-0,308^{+} \\
(0,1721) \\
{[0,1650]}\end{array}$ & $\begin{array}{c}0,0078 \\
(0,1078) \\
{[0,1122]}\end{array}$ & $\begin{array}{c}0,2028 \\
(0,2207) \\
{[0,2175]}\end{array}$ \\
\hline Fachhochschulreife & $\begin{array}{c}0,1844 \\
(0,1886) \\
{[0,2094]}\end{array}$ & $\begin{array}{c}0,2780 \\
(0,3493) \\
{[0,3497]}\end{array}$ & $\begin{array}{l}-0,1555 \\
(0,1966) \\
{[0,1654]}\end{array}$ & $\begin{array}{c}-0,4278 \\
(0,3807) \\
{[0,3564]}\end{array}$ \\
\hline Abitur & $\begin{array}{c}0,2602^{*} \\
(0,1504) \\
{[0,1329]}\end{array}$ & $\begin{array}{c}0,1422 \\
(0,2920) \\
{[0,3101]}\end{array}$ & $\begin{array}{l}0,3012^{+} \\
(0,1377) \\
{[0,1597]}\end{array}$ & $\begin{array}{c}0,2335 \\
(0,3035) \\
{[0,2470]}\end{array}$ \\
\hline Kein Abschluss & $\begin{array}{l}-0,2450 \\
(0,2083) \\
{[0,1972]}\end{array}$ & $\begin{array}{l}-0,3139 \\
(0,2344) \\
{[0,2369]}\end{array}$ & $\begin{array}{c}0,3009^{+} \\
(0,1918) \\
{[0,1549]}\end{array}$ & $\begin{array}{c}0,4735 \\
(0,4201) \\
{[0,3364]}\end{array}$ \\
\hline \multicolumn{5}{|c|}{$\begin{array}{l}\text { Stellung im Beruf (Basis = Ange- } \\
\text { stellte(r) mit qualifizierter Tätigkeit) }\end{array}$} \\
\hline Ungelernte(r) Arbeiter(in) & $\begin{array}{c}-0,6906^{* *} \\
(0,2473) \\
{[0,2472]}\end{array}$ & $\begin{array}{l}-0,2655 \\
(0,2787) \\
{[0,2656]}\end{array}$ & $\begin{array}{l}-0,0868 \\
(0,2010) \\
{[0,1934]}\end{array}$ & $\begin{array}{c}0,0059 \\
(0,3166) \\
{[0,3236]}\end{array}$ \\
\hline Angelernte(r) Arbeiter(in) & $\begin{array}{c}-0,2763^{+} \\
(0,1624) \\
{[0,1429]}\end{array}$ & $\begin{array}{c}0,0627 \\
(0,2312) \\
{[0,1880]}\end{array}$ & $\begin{array}{c}0,0839 \\
(0,1596) \\
{[0,1717]}\end{array}$ & $\begin{array}{r}-0,5793^{+} \\
(0,2991) \\
{[0,3024]}\end{array}$ \\
\hline Gelernte(r) Facharbeiter(in) & $\begin{array}{c}0,0023 \\
(0,2378) \\
{[0,1249]}\end{array}$ & $\begin{array}{l}-0,0316 \\
(0,2286) \\
{[0,2239]}\end{array}$ & $\begin{array}{c}0,2619 \\
(0,1743) \\
{[0,1686]}\end{array}$ & $\begin{array}{c}-0,1652 \\
(0,4219) \\
{[0,4177]}\end{array}$ \\
\hline
\end{tabular}

Fortsetzung auf der nächsten Seite 
Fortsetzung 2 von Tabelle 4:

\begin{tabular}{|c|c|c|c|c|}
\hline \multirow[t]{2}{*}{ Variable } & \multicolumn{2}{|c|}{ Männer } & \multicolumn{2}{|c|}{ Frauen } \\
\hline & $\mathrm{K}$ & $\mathrm{E}$ & $\mathrm{K}$ & $\mathrm{E}$ \\
\hline \multirow[t]{3}{*}{ Vorarbeiter(in)/Meister(in) } & 0,2793 & $0,6885^{+}$ & $-0,0479$ & - \\
\hline & $(0,2166)$ & $(0,3902)$ & $(0,4870)$ & - \\
\hline & {$[0,2321]$} & {$[0,3921]$} & {$[0,3646]$} & - \\
\hline \multirow[t]{3}{*}{ Angestellte(r) mit einfacher Tätigkeit } & $-0,3131^{+}$ & $-0,6776^{*}$ & $-0,0926$ & 0,1496 \\
\hline & $(0,1800)$ & $(0,2918)$ & $(0,1112)$ & $(0,2381)$ \\
\hline & {$[0,1639]$} & {$[0,2670]$} & {$[0,1121]$} & {$[0,1795]$} \\
\hline \multirow[t]{3}{*}{ Angestellte(r) mit hochqual. Tätigkeit } & 0,0046 & 0,1199 & $-0,3796^{*}$ & $-0,1301$ \\
\hline & $(0,1416)$ & $(0,2893)$ & $(0,1765)$ & $(0,2927)$ \\
\hline & {$[0,1430]$} & {$[0,2677]$} & {$[0,1843]$} & {$[0,2637]$} \\
\hline \multirow[t]{3}{*}{ Teilzeit } & $-0,0981$ & $-0,4054$ & $-0,1635^{+}$ & $-0,2679$ \\
\hline & $(0,2555)$ & $(0,5126)$ & $(0,1024)$ & $(0,2260)$ \\
\hline & {$[0,2681]$} & {$[0,4806]$} & {$[0,0843]$} & {$[0,2003]$} \\
\hline \multirow[t]{3}{*}{ Öffentlicher Dienst } & $-0,3973^{*}$ & $-0,8792^{* *}$ & $-0,1233$ & $-0,4297^{+}$ \\
\hline & $(0,1688)$ & $(0,2887)$ & $(0,1272)$ & $(0,2778)$ \\
\hline & {$[0,1685]$} & {$[0,2657]$} & {$[0,1090]$} & {$[0,2532]$} \\
\hline \multicolumn{5}{|l|}{$\begin{array}{l}\text { Firmengröße (Basis = } 20 \text { - } 199 \text { Be- } \\
\text { schäftigte) }\end{array}$} \\
\hline \multirow[t]{3}{*}{1 - 19 Beschäftigte } & $-0,0825$ & 0,1620 & $-0,0537$ & $-0,4118^{+}$ \\
\hline & $(0,1732)$ & $(0,2179)$ & $(0,1471)$ & $(0,2541)$ \\
\hline & {$[0,1666]$} & {$[0,2237]$} & {$[0,1433]$} & {$[0,2284]$} \\
\hline \multirow[t]{3}{*}{200 - 1999 Beschäftigte } & $-0,5277^{*}$ & $-0,4962^{+}$ & $-0,3928^{+}$ & $1,0987^{+}$ \\
\hline & $(0,2739)$ & $(0,3473)$ & $(0,2672)$ & $(0,6911)$ \\
\hline & {$[0,2415]$} & {$[0,2842]$} & {$[0,2211]$} & {$[0,6309]$} \\
\hline \multirow[t]{3}{*}{2000 und mehr Beschäftigte } & $-0,1102$ & $-0,1269$ & 0,1127 & 0,0358 \\
\hline & $(0,0938)$ & $(0,1435)$ & $(0,1002)$ & $(0,2102)$ \\
\hline & {$[0,0996]$} & {$[0,1466]$} & {$[0,0902]$} & {$[0,2183]$} \\
\hline \multicolumn{5}{|l|}{$\begin{array}{l}\text { Wirtschaftssektor (Basis = Produ- } \\
\text { zierendes Gewerbe) }\end{array}$} \\
\hline 4 Wirtschaftsdummies & ja & ja & ja & ja \\
\hline Zeittrend & ja & ja & ja & ja \\
\hline \multirow[t]{3}{*}{ Konstante } & $4,0733^{* *}$ & $3,3831^{* *}$ & $3,4057^{* *}$ & $3,0350^{* *}$ \\
\hline & $(0,2378)$ & $(0,3878)$ & $(0,2300)$ & $(0,3974)$ \\
\hline & {$[0,2281]$} & {$[0,3705]$} & {$[0,1754]$} & {$[0,4045]$} \\
\hline \multicolumn{5}{|c|}{$\begin{array}{c}\text { K: Kündigungen E: Entlassungen } \\
\text { Koeffizienten-/Parameterschätzungen basierend auf Gleichung (1) } \\
\text { Heteroskedastierobuste Standardfehler in runden Klammern } \\
\text { Heteroskedastie- und clusterrobuste Standardfehler in eckigen Klammern } \\
{ }^{+} ;^{*} \text { bzw. }{ }^{* *} \text { zeigt Signifikanz der Koeffizienten auf einem Niveau von } \alpha=0,1 ; 0,05 \text { bzw. } 0,01 \\
\text { Bei den Frauen musste in der Schätzung für Entlassungen aufgrund geringer Fallzahlen auf } \\
\text { den Einschluss der Variablen "Vorarbeiterin/Meisterin" verzichtet werden. }\end{array}$} \\
\hline
\end{tabular}

